\title{
Finite-connectivity spin-glass phase diagrams and low-density parity check codes
}

\author{
Gabriele Migliorini and David Saad \\ The Neural Computing Research Group, School of Engineering and Applied Sciences, Aston University, Birmingham B4 7ET, \\ United Kingdom \\ (Received 20 July 2005; published 21 February 2006)
}

\begin{abstract}
We obtain phase diagrams of regular and irregular finite-connectivity spin glasses. Contact is first established between properties of the phase diagram and the performance of low-density parity check (LDPC) codes within the replica symmetric (RS) ansatz. We then study the location of the dynamical and critical transition points of these systems within the one step replica symmetry breaking theory (RSB), extending similar calculations that have been performed in the past for the Bethe spin-glass problem. We observe that the location of the dynamical transition line does change within the RSB theory, in comparison with the results obtained in the RS case. For LDPC decoding of messages transmitted over the binary erasure channel we find, at zero temperature and rate $R=1 / 4$, an RS critical transition point at $p_{c} \simeq 0.67$ while the critical RSB transition point is located at $p_{c} \simeq 0.7450 \pm 0.0050$, to be compared with the corresponding Shannon bound $1-R$. For the binary symmetric channel we show that the low temperature reentrant behavior of the dynamical transition line, observed within the RS ansatz, changes its location when the RSB ansatz is employed; the dynamical transition point occurs at higher values of the channel noise. Possible practical implications to improve the performance of the state-ofthe-art error correcting codes are discussed.
\end{abstract}

DOI: $10.1103 /$ PhysRevE.73.026122

PACS number(s): 89.90.+n, 89.70.+c, 05.50.+q

\section{INTRODUCTION}

The persistence of ferromagnetic ordering under the disruption of frozen random fields [1] and the onset of spinglass (SG) order in systems characterized by random competing interactions [2] are two central problems in the statistical mechanics of systems with quenched randomness. When properly rephrased, these two problems turn out to relate to core problems in other, quite different disciplines. Insight into these two closely related problems for any given system is obtained from the corresponding phase diagram as a function of the physical parameters, such as temperature and disorder strength.

Finite-connectivity mean-field spin models [3-7] and their $p$-spin counterpart [8] are important for two distinct reasons. First, despite being mean-field in nature, they are believed to share common properties with finite-dimensional spin-glass systems $[9,10]$. Second, the statistical mechanics of SG model systems with fixed finite connectivity relates to the study of various hard computational problems. In the last two decades the analysis of finite-connectivity spin systems has also offered new tools for understanding the very nature of several optimization problems and improve algorithmic performances $[11,12]$. In this paper we first obtain the phase diagram of finite-connectivity mean-field spin models. We then investigate the relation between features of the phase diagram and the performance of low-density parity check (LDPC) codes, analyzing the effects induced by the replica symmetry broken (RSB) nature of solutions obtained for these finite-connectivity spin models [13]. In order to answer these questions, we exploit the relation between finiteconnectivity spin systems and a class of exactly solvable models; we employ an efficient computational method that has been shown to be effective for understanding several features of finite-dimensional SG [14].

We will consider a generalized model unifying the frameworks for a few distinct problems. Particular attention will be given to two special cases: (i) The Bethe SG which has been extensively studied under both replica symmetric (RS) and RSB Ansätze [3-5] and recently considered in its ferromagnetically biased version [15]; (ii) Gallager (LDPC) errorcorrecting codes [16]. While the former is well known within the statistical physics community, many in this community are less familiar with the latter and its links to the physics of disordered systems.

Reliable transmission of information in noisy conditions is one of the basic problems of modern communication technology. Correspondingly, one of the central problems of information theory is channel coding. Methods to achieve reliable information transmission rely on the introduction of structured redundancy to the original message in order to compensate for corruption due to noise. Shannon derived rigorous bounds on the level of redundancy (the code rate) required to enable error-free communication for a given channel noise [17]. However, Shannon's theorems are nonconstructive and do not offer a practical coding scheme. Different methods, based on various redundancy construction recipes, have been proposed over the years [18]. Despite the fact that error-correcting codes are now widely used in a variety of applications, current performances of most methods are significantly below Shannon's bound. One family of error-correcting codes that have been shown to provide close-to-optimal performance is that of Gallager's LDPC codes [16]. In these codes, structured redundancy is introduced through parity checks of Boolean sums of randomly sampled message bits. The relation between the parity-check error-correcting codes and spin models, and consequently between quite different disciplines as statistical physics and information theory, has been pointed to in the seminal work of Sourlas [19].

Decoding in LDPC codes corresponds to a class of spin models defined by an underlying geometry that reflects the low density character of the code construction. This very last 
point, that made the above correspondence useful and of practical relevance, was investigated recently in Ref. [20] and in a number of subsequent publications. Methods developed in the statistical mechanics of diluted spin models under the RS Ansatz have been successfully employed to compute macroscopic properties of such systems for different parameter values; good agreement with observed decoding bounds have been reported [21]. To our knowledge, however, the only phase diagram that has been presented for LDPC codes is in the limit of large coordination-large multispin interaction numbers [22] (although the expressions themselves have been derived for the general case), which also relates to the phase diagram of the random energy model (REM) [23].

In the following section we introduce the class of Ising spin systems to be analyzed and its links to the Bethe SG and LDPC decoding problems. This will be followed by Sec. III where we review the RS equations and the corresponding solutions obtained using a computational method adopted from the study of $d$-dimensional hierarchical models. As part of this section we will present the method and briefly discuss the relation between finite-connectivity SG and $d$-dimensional hierarchical models. In Sec. IV we introduce the equations behind the RSB theory of finite-connectivity SG studied here and present results obtained for the Bethe SG problem and LDPC error-correcting codes under two different transmission channels: the binary erasure channel (BEC) and the binary symmetric channel (BSC). In Sec. V we discuss the results obtained and point to future research directions.

\section{MODEL}

The two different models examined in this work stem from a unified physical system of $K$-spin interaction Ising model on a lattice of fixed connectivity $C$. The Hamiltonian describing the system takes the form

$$
\mathcal{H}_{0}=-J \sum_{\mu} \mathcal{A}_{\mu} \prod_{i \in \mathcal{L}(\mu)} S_{i},
$$

where $S \in\{ \pm 1\}^{N}$ represents $N$ binary variables, $\mathcal{L}(\mu)$ $=\left\langle i_{1}, \ldots, i_{K}\right\rangle$ is a set of indices related to interaction index $\mu$ and $\mathcal{A}_{\mu}$ is the corresponding connectivity tensor with the following properties:

$$
\begin{gathered}
\mathcal{A}_{\left\langle i_{1}, \ldots, i_{K}\right\rangle}^{\mu}= \begin{cases}1 \text { for } & \mathcal{L}(\mu)=\left\langle i_{1}, \ldots i_{K}\right\rangle \\
0 & \text { otherwise }\end{cases} \\
\sum_{\left\langle i_{2}, \ldots, i_{K}\right\rangle} \mathcal{A}_{\left\langle i, i_{2}, \ldots, i_{K}\right\rangle}=C \forall i .
\end{gathered}
$$

The parameter $J$ is related to the strength of the random exchange interaction. The various models considered in this work differ in the connectivity parameters and type of bias used, which corresponds to an additional component of the Hamiltonian (1).

\section{A. Bethe SG}

The Bethe SG has been extensively studied in the past [3-5] and represents a special case of the Hamiltonian (1) where one is only considering pairwise interactions, $K=2$.

Bethe SG with ferromagnetically biased exchange interaction $J$, which we will focus on here, has been recently studied in Ref. [15], where the distribution of the quenched random variable $J$ takes the form

$$
P(J)=\frac{1+\rho}{2} \delta(J-1)+\frac{1-\rho}{2} \delta(J+1),
$$

and where the parameter $\rho \in[0,1]$ varies between $\rho=1$ for a ferromagnet and $\rho=0$ for the unbiased Bethe SG.

\section{B. LDPC error-correcting codes}

We consider a decoding problem in a communication model where $L$ bit messages are encoded using LDPC codes and then communicated through two channel types: (i) the binary symmetric channel (BSC) where bits are flipped with probability $p$ during transmission, and (ii) the binary erasure channel (BEC) where bits are received uncorrupted with probability $1-p$ and fail to arrive with probability $p$ (i.e., no information is provided for these).

The analogy between the communication model and spin models is straightforward, transmitted message bits correspond to spin variables and the structure defined by coding system corresponds to interactions between them, as will be explained below. Before introducing the statistical physics representation of the decoding problem we briefly introduce the main concepts behind LDPC error-correcting codes and review the characteristics of two communication channels examined in this work.

A Gallager LDPC code is defined by a binary matrix $\boldsymbol{H}=[\boldsymbol{A} \mid \boldsymbol{B}]$, concatenating two very sparse matrices known to both sender and receiver, with $\boldsymbol{B}$ [of dimensionality $(N-L)$ $\times(N-L)]$ and invertible, while the matrix $\boldsymbol{A}$ is of dimensionality $(N-L) \times L$. Both matrices are characterized by a few number of unit elements per row and column, which is much lower than the dimensionality of the matrices. LDPC codes can be employed over various finite fields [24]; we will restrict the treatment in this paper to binary values.

Encoding refers to mapping the original $L$ dimensional binary message vector $\boldsymbol{\xi} \in\{0,1\}^{L}$ to an $N$ dimensional binary code word $\boldsymbol{t} \in\{0,1\}^{N}(N>L)$ by taking the product $\boldsymbol{t}=\boldsymbol{G}^{\boldsymbol{T}} \boldsymbol{\xi}$ $(\bmod 2)$, where all operations are performed in the field $\{0,1\}$ and are indicated by $(\bmod 2)$. The generator matrix is $\boldsymbol{G}=\left[\boldsymbol{I} \mid\left(\boldsymbol{B}^{-1} \boldsymbol{A}\right)^{T}\right](\bmod 2)$, where $\boldsymbol{I}$ is the $L \times L$ identity matrix, implying that $\boldsymbol{H G}^{\boldsymbol{T}}(\bmod 2)=0$ (identically, by the suggested construction) and that the first $L$ bits of $\boldsymbol{t}$ are set to the message $\boldsymbol{\xi}$ vector itself. In regular Gallager codes the number of nonzero unit elements in each row of $\boldsymbol{H}$ is chosen to be exactly $K$. The number of elements per column is then $C$ $=(1-R) K$, where the code rate $R=L / N$ represents the fraction of informative bits (for unbiased messages). Both $K$ and $C$ are finite and of $O(1) \ll O(N)$, while both $L$ is of $O(N)$ and the rate $R$ of $O(1)$. We will mostly focus here on regular code constructions, where the system size is infinite.

As will be shown below, the sparse matrix $\boldsymbol{H}$ gives rise to the interaction between noise (BSC) or codeword (BEC) bits that can be represented by spin variables. The decoding pro- 
cess corresponds to finding the ground state of the corresponding Ising spin model.

\section{BSC}

The corruption process that is most intuitively linked to physical systems is that of the BSC, where the encoded vector $\boldsymbol{t}$ is corrupted by flip noise represented by the vector $\zeta \in\{0,1\}^{L}$ with components independently drawn from $P(\zeta)=(1-p) \delta(\zeta)+p \delta(\zeta-1)$. The noise flips give rise to ferromagnetic and non-ferromagnetic interactions between the system variables. The received vector takes the form $\boldsymbol{r}$ $=\boldsymbol{G}^{\boldsymbol{T}} \boldsymbol{\xi}+\boldsymbol{\zeta}(\bmod 2)$.

Decoding is carried out by multiplying the received message by the matrix $\boldsymbol{H}$ to produce the syndrome vector $\boldsymbol{z}=\boldsymbol{H} \boldsymbol{r}=\boldsymbol{H} \boldsymbol{\zeta}(\bmod 2)$ from which an estimate $\hat{\boldsymbol{\zeta}}$ for the noise vector can be inferred. An estimate for the original message is then obtained as the first $L$ bits of $\boldsymbol{r}+\hat{\boldsymbol{\zeta}}(\bmod 2)$.

The Bayes optimal symbol estimator [also known as Marginal Posterior Maximizer (MPM)] for noise vector bits is defined as the variable value that maximizes the corresponding marginal probability $\hat{\zeta}_{j}=\operatorname{argmax}_{S_{j}} P\left(S_{j} \mid z\right)$, where $S$ represent a candidate noise variable taking the values $\{0,1\}$. Computing the MPM estimate directly is computationally hard, and is typically approximated in practice by employing an iterative inference algorithm such as belief propagation [25]. This and similar iterative inference algorithms can be directly related to mean-field methods in general and the Bethe approximation in particular [26,27].

The connection to statistical physics becomes clear when the field $\{0,1\}$ is replaced by Ising spins $\{ \pm 1\}$ and $\bmod 2$ sums by products [19]. The syndrome vector acquires the form of a multispin coupling $\mathcal{J}_{\mu}=\Pi_{i \in \mathcal{L}(\mu)} \zeta_{i}$ where $i=1, \ldots, N$ and $\mu=1, \ldots,(N-L)$. The $K$ indices of nonzero elements in the row $\mu$ of $\boldsymbol{A}$ are given by $\mathcal{L}(\mu)=\left\{i_{1}, \ldots, i_{K}\right\}$ and where $\mathcal{A}_{\left\langle i_{1}, \cdots i_{K}\right\rangle}$ is the connectivity tensor, given in Eq. (2). After gauging $S_{j} \rightarrow S_{j} \zeta_{j}$ the multispin coupling coefficient vanishes $\left(\mathcal{J}_{\mu}=1\right)$ and the parity check component of the Hamiltonian takes the form (1) with $J \rightarrow \infty$ forcing all candidate vectors to obey the parity checks [which, in the $\{0,1\}$ representation correspond to the equation $\boldsymbol{z}=\boldsymbol{H} \boldsymbol{S}(\mathrm{mod}$ 2)]. This notation is used to distinguish the temperature from the parity check constraints and to facilitate the finite temperature study of error-correcting codes.

Prior knowledge of the biased noise vector is added through a second component to the Hamiltonian (1). After gauging, the new term takes the form $H_{1}=-F \sum_{j=1}^{N} S_{j} \zeta_{j}$ where $F=\frac{1}{2} \ln [(1-p) / p]$ is the Nishimori condition $[28,29]$ (with $\beta=1$ ), which represents the correct prior for the variables $\boldsymbol{S}$. Decoding at the Nishimori condition corresponds to the MPM estimation [30]. This gives rise to the Hamiltonian

$$
-\beta \mathcal{H}=\beta J \sum_{\mu} \mathcal{A}_{\mu} \prod_{i \in \mathcal{L}(\mu)} S_{i}+\beta F \sum_{j=1}^{N} S_{j} \zeta_{j}
$$

for the BSC.

Another important estimator one should mention is the Maximum A Posteriori (MAP) estimator defined as
$\hat{\boldsymbol{\zeta}}=\operatorname{argmax}_{S} P(\boldsymbol{S} \mid z)$. This corresponds to zero temperature decoding [30].

\section{2. $B E C$}

Codeword bits transmitted over a BEC have a probability $1-p$ of being received (in tact). Encoding is carried out similarly to the BSC, but decoding is based on inferring the codeword itself, rather than the noise vector.

In the case of the BEC, solution vectors are by definition codewords and should therefore obey $\boldsymbol{H} \boldsymbol{S}(\bmod 2)=0$, where $S$ are the received vectors (codewords with a certain number of unknown bits).

An estimate of the original message is then obtained by inferring the missing bits using MPM. Similar practical methods to those described in the BSC may be employed also in this case.

The statistical mechanics formulation of the problem is similar to that of the BSC, except for the second component added to the Hamiltonian (1), that takes the form

$$
\mathcal{H}_{1}=-\sum_{i=1}^{N} \ln \left[p+(1-p) \delta_{S_{i}, 1}\right]
$$

Code performance in the BEC will be considered only at the zero temperature limit as in Ref. [31].

A review of LDPC codes and their link to statistical mechanics has been presented in Ref. [32].

\section{REPLICA SYMMETRIC THEORY}

The analysis carried out here is a natural extension of similar calculations done in the past for the Bethe SG [33] model and $p$-spin Ising models [3]. We therefore briefly review the RS theory of the model described by Hamiltonian (4) as both the Bethe SG and LDPC decoding can be considered as special cases of the same model.

To compute the free energy of the problem, consider the replica equality,

$$
\beta f=-\left.\lim _{N \rightarrow \infty} \frac{1}{N} \frac{\partial}{\partial n}\right|_{n \rightarrow 0}\left\langle\mathcal{Z}^{n}\right\rangle_{\mathcal{A}, \zeta, J},
$$

where $\left\langle\mathcal{Z}^{n}\right\rangle_{\mathcal{A}, \zeta, J}$ is the analytical continuation in the interval $n \in[0,1]$ of the replicated partition function [34]. Following the standard derivation, e.g., as in Ref. [21,35], and employing the RS Ansatz, one obtains the following expression for the replica symmetric free energy as a function of the local field distribution $\pi(x)$ (details can be found in Appendix A):

$$
\beta f=\Delta f^{(1)}-C \frac{K-1}{K} \Delta f^{(2)} .
$$

There are both site and bond contributions,

$$
\Delta f^{(1)}=-E_{J, \zeta} \int \prod_{j=1}^{C} \prod_{j^{\prime}=1}^{K-1} d x_{j j^{\prime}} \pi\left(x_{j j^{\prime}}\right) \mathcal{F}^{(1)}\left(\left\{x_{j j^{\prime}}\right\}, \zeta, J_{j}\right),
$$




$$
\Delta f^{(2)}=-E_{J} \int \prod_{j=1}^{K} d x_{j} \pi\left(x_{j}\right) \mathcal{F}^{(2)}\left(\left\{x_{j}\right\}, J_{j}\right),
$$

where $E_{J, \zeta}$ denotes an average over the two sources of randomness in Eq. (4), and

$$
\begin{gathered}
\mathcal{F}_{a}^{(1)}=\ln 2 \cosh \beta\left(\sum_{j} u\left(\left\{x_{j j^{\prime}}\right\}, J_{j}\right)+F \zeta\right), \\
\mathcal{F}_{b}^{(1)}=a\left(\left\{x_{j j^{\prime}}\right\}, J_{j}\right), \\
\mathcal{F}^{(2)}=a\left(\left\{x_{j}\right\}, J_{j}\right)+u\left(\left\{x_{j}\right\}, J_{j}\right) .
\end{gathered}
$$

We denote $\mathcal{F}^{(1)}=\mathcal{F}_{a}^{(1)}+\mathcal{F}_{b}^{(1)}$ where

$$
\begin{aligned}
& \beta u\left(\left\{x_{j}\right\}, J\right)=\frac{1}{2} \ln [R(+) / R(-)] \\
& \beta a\left(\left\{x_{j}\right\}, J\right)=\frac{1}{2} \ln [R(+) R(-)] .
\end{aligned}
$$

The polynomials $R(\sigma)$ are defined via

$$
R(\sigma)=\sum_{\sigma_{1}, \cdots \sigma_{K-1}} \exp \beta\left[y_{1} \sigma \sigma_{1}+y_{2} \sigma_{1} \sigma_{2}+\cdots y_{K} \sigma_{K-1} \sigma\right],
$$

where $\left\{y_{1}, \ldots, y_{K}\right\} \equiv\left\{x_{1}, \ldots x_{K-1}, J\right\}$. The above expression, in a similar form, has already been obtained in Ref. [20], and the equivalence with their expression is shown in Appendix A. We prefer the current formulation because of its manifest simplicity (the two contributions to the free energy coming from sites and bonds are here in evidence) and because it depends on the function $a\left(\left\{x_{j}\right\}, J_{j}\right)$, which will play a central role in the RSB case, examined in Sec. IV.

The above expression also introduces the polynomials $R(\sigma)$, already well known in the context of hierarchical lattices [14]. Evaluating the saddle point equations of the above free-energy expression is the basic premise of the RS theory, so that the crux of the quenched randomness problem (4) lies in the convolution [20]

$$
\pi(x)=E_{J, \zeta} \int \prod_{i j} d x_{i j} \pi\left(x_{i j}\right) \delta\left(x-\mathcal{R}\left(\left\{x_{i j}\right\}, J, \zeta\right)\right),
$$

where $i=1, \ldots, C-1, j=1, \ldots, K-1$; and where the recursion relations are defined via

$$
\mathcal{R}\left(\left\{x_{i j}\right\}, J, \zeta\right)=\sum_{j=1}^{C-1} u\left(\left\{x_{i j}\right\}, J_{j}\right)+F \zeta .
$$

We can also rewrite for convenience the above convolution with the aid of the auxiliary distribution $\hat{\pi}(\hat{x})$, as a set of two coupled equations (cf. Appendix A),

$$
\begin{gathered}
\pi(x)=E_{\zeta} \int \prod_{i=1}^{C-1} d \hat{x}_{i} \hat{\pi}\left(\hat{x}_{i}\right) \delta\left(x-\sum_{i=1}^{C-1} \hat{x}_{i}-F \zeta\right), \\
\hat{\pi}(\hat{x})=E_{J} \int \prod_{j=1}^{K-1} d x_{j} \pi\left(x_{j}\right) \delta\left(\hat{x}-u\left(\left\{x_{j}\right\}, J\right)\right) .
\end{gathered}
$$

\section{A. Saddle point equations and LDPC decoding}

To briefly explain the link between LDPC decoding and the replica formulation we will restrict this discussion to the BSC. The replica analysis as well as decoding in other noisy channels follow a similar path [36].

Following the derivation of Ref. [21] we write saddle point equations for the LDPC decoding problem, considering a special case of the Hamiltonian (4) and the saddle point equations (14), where $J \rightarrow \infty$ is deterministic,

$$
\pi(x)=E_{\zeta} \int \prod_{i=1}^{C-1} d \hat{x}_{i} \hat{\pi}(\hat{x}) \delta\left(x-\sum_{i=1}^{C-1} \hat{x}_{i}-F \zeta\right),
$$

$$
\hat{\pi}(\hat{x})=\int \prod_{j=1}^{K-1} d x_{j} \pi\left(x_{j}\right) \delta\left[\hat{x}-u_{\beta}\left(\left\{x_{j}\right\}\right)\right] .
$$

Most of the numerical results presented so far were obtained along the Nishimori line $\beta=1$. Here we investigate the phase diagram at any prior $1 / \beta F$, for different choices of the parameters $C$ and $K$. A typical case we will discuss in detail is the one corresponding to $C=3, K=4$.

We will check the consistency between the results obtained and actual decoding experiments, using belief propagation (BP) [37], at and away from the Nishimori condition. We will not review BP in the paper as it is a well known algorithm $[25,38]$ with strong links to the Bethe approximation and its variants $[26,27]$. The iterative BP algorithm, in the context of LDPC decoding, is a microscopic equivalent of Eqs. (15).

\section{B. Computational method}

The set of (RS) equations (14) have been considered and solved in a variety of different ways already. This section introduces a different method. We propose an alternative approach to existing methods (e.g., population dynamics or Monte Carlo techniques) which solves equations of the form (12) efficiently and accurately. The main reason it has not been used to study finite-connectivity spin models so far is probably due to the different context in which it was first introduced [39]. The method we propose allows us to determine the phase diagram of diluted systems of the type studied here and analyze the nature and exact location of the phase boundaries. It borrows tools and concepts from a different branch of research, namely the area of hierarchical models $[14,40]$, to obtain accurate phase diagrams. The iterative method we present here has a corresponding computational effort that scales as $\mathrm{N}^{K-1}, \mathrm{~N}$ being the number of bins introduced and $K$ the multispin interaction parameter.

Equation (12) complicates, after a few iterations, even a simple initial local field distribution. The distribution $\pi(x)$ and its conjugate $\hat{\pi}(\hat{x})$ are represented via histograms. Each histogram is characterized by two quantities, $x_{i j}$ and $\pi\left(x_{i j}\right)$, where the latter is the associated probability. Equation (15) is the convolution of $(K-1) C$ distributions, taking into account the distribution of the random field $P(\zeta)$, contained in the average $E_{\zeta}(\cdots)=\int P(\zeta) d \zeta(\cdots)$. This is numerically constructed from pairwise convolutions of distributions. For an 
$R=1 / 4$ rate Gallager code, e.g., three pairwise convolutions are required. A pairwise convolution (or binary operation) is achieved as follows:

(i) Histogram representation: The histograms are represented by a properly drawn grid at each iteration step of the algorithm. All histogram values that fall within the same grid cell are combined in such a way as to preserve the averages across the domain of the local field (and its conjugated) distribution(s). Histogram values that fall outside the grid, representing a pre-determined small fraction of the overall probability, are similarly combined into a single histogram cell. In this procedure, a histogram that falls within a narrow band of a grid boundary is proportionally shared between neighboring bins. The gridding is done separately for $x>0$ and $x<0$.

(ii) Pairwise convolution: Two gridded distributions are convoluted according to Eq. (14) regenerating the original number of histograms.

(iii) Multiple convolutions: The convolution of $C(K-1)$ distributions is achieved, from pairwise convolutions, as follows: (a) The pairwise convolution contained in the first equation of Eq. (14) is cycled once for, e.g., a code rate $R=1 / 4$. (b) The resulting distribution is pairwise-convoluted with the noise channel distribution $P(\zeta)$. (c) The resulting distribution is further self-convoluted according to the second equation in Eq. (14). This completes the entire process, that is then iterated until convergence is achieved. The flow of the probability distribution $\pi(x)$ determines the phase diagram of the system. Most of our results have been obtained considering $125 \times 4$ independent bins, corresponding to the flows of $10^{3}$ quantities (the corresponding number of elementary operations considered is the number of bins to the power of $K-1$ ). Each of the two grids at $x>0$ and $x<0$ have, e.g., 125 bins on each side of the average value.

A similar set of equations to Eq. (12) have been discussed in a variety of different contexts. A very important one is that of real-space renormalization-group theory [39-41].

Although the algebraic form of the convolution corresponding to these two problems is the same, the two problems are indeed very different. In the case of hierarchical models the distribution of the exchange interaction is studied, while in the current problem one considers the distribution of the local field. This reflects the difference in the two underlying geometries. The $d$-dimensional hierarchical lattice includes loops that are an essential ingredient in making it a better candidate for studying the problem of finitedimensional SG [14] (within exactly solvable models). The similarity between Eqs. (15) and those related to hierarchical models is indicative of a deeper relation between the two (both geometries can be considered as hierarchical [42]), which will be the subject of future work.

\section{LDPC phase diagram-BSC}

The calculated phase diagram for the Gallager LDPC code of rate $R=1 / 4$ and a BSC of flip rate $p$ as a function of the prior temperature $1 / \beta F$ are shown in Fig. 1. We report both the location of the dynamical noise transition $p_{d}$, defined by the noise level at which suboptimal solutions

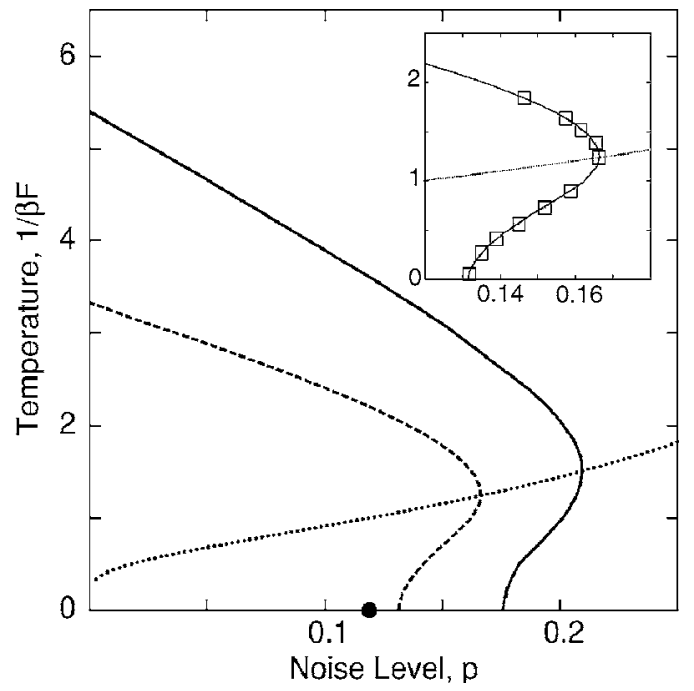

FIG. 1. The phase diagram for the LDPC code of rate $R=1 / 4$, where codewords are transmitted over the BSC. The solid and dashed lines represent the critical and dynamical transitions, respectively. The inset shows the curvature of the dynamical transition line boundary, together with decoding experiments results (square boxes, variance smaller than symbol size). The dotted curve represents the Nishimori line. The black filled dot indicates the location of the dynamical transition at zero temperature assuming the local field distribution to be peaked at integer values only.

emerge, and critical noise transition $p_{c}$, where the ferromagnetic solution ceases to be dominant, for all ranges of rescaled temperature $1 / \beta F$. The phase diagram was calculated considering the flows of $10^{3}$ quantities dictated by the convolution (12). As mentioned before this number should be taken to the power $K-1$ to obtain the number of elementary operations within, e.g., the second equation of Eq. (14).

The location of the dynamical and critical transition values can be determined by analyzing the free energy and the complexity as functions of the channel noise level for different values of the temperature $1 / \beta$ (Fig. 2). At any value $p<p_{d}$, the computed free energy (8) reduces to the ferromagnetic free energy $f=-(1-2 p) F$. Consider first temperature values in the proximity of the Nishimori line. The trajectories exhibit a crossover to an unstable distribution first, and then flow to the ferromagnetic sink below $\simeq 0.1665$ (corresponding to a decoding success) or to a stable nonferromagnetic distribution (corresponding to a decoding failure) above it. A similar behavior is observed for any temperature above the Nishimori line, the stable distribution being different for each value of the temperature $\beta<1$. Rather different behavior is observed below the Nishimori line, where the unstable distribution is a strong coupling distribution, meaning that the average local field strength increases but the rescaled distribution remains constant. Finally, the trajectory flows again, either to the ferromagnetic sink or to a fixed local field distribution, which is stable under iteration of the recursion relation (15).

The computed free energy, according to the convolution (15), is higher than the ferromagnetic free energy for all noise levels $p_{d}<p<p_{c}$. Above the critical noise value $p_{c}$, the computed free energy becomes lower than the ferromagnetic 


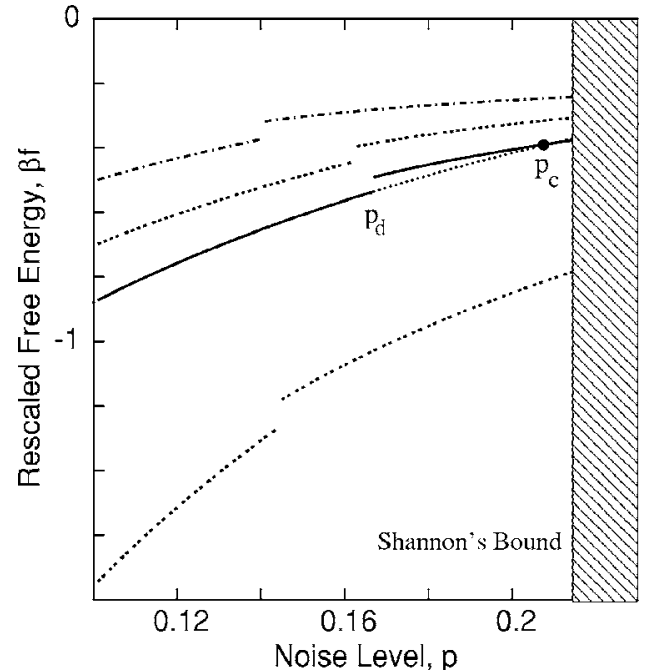

FIG. 2. The computed free energy (8) as a function of the noise level $p$ for different values of $\beta$. The solid line corresponds to the free energy at the Nishimori line $\beta=1$, while the dashed lines correspond to the values of $\beta=8 / 4$ below the Nishimori line and $\beta$ $=8 / 10,8 / 14$ above it. For flip rates $p$ larger than the dynamical transition the computed free energy is higher than the ferromagnetic one, given by $f_{\text {Ferro }}=-(1-2 p) F$ (dotted line).

free energy. The location of the dynamical transition has been determined, at any temperature, by the nature of the flow of $\pi(x)$ under iteration of the convolution (12), i.e., whether it flows to the ferromagnetic solution or otherwise. The location of the critical noise threshold has been determined from the free energy (8) as the point where the computed and the ferromagnetic free energies coincide as shown in Fig. 2. In the inset of Fig. 1 we also report (square boxes) the results of real decoding experiments obtained as follows: We iterated the probabilistic decoding algorithm [25], at different values of the channel noise $p$, for an ensemble of $10^{3}$ networks each of $10^{4}$ and $2 \times 10^{4}$ nodes, respectively. The inset shows the calculated mean values, the corresponding error being smaller than the symbol size. A precise estimate of the dynamical transition point has been obtained considering the percentage of decoding success within the ensemble as a function of the noise level, for different system sizes [43]. The decoding experiments were performed for different choices of the temperature. Along the Nishimori line (see Fig. 1) we find the value of $p_{d} \simeq 0.1665 \pm 0.0005$ in excellent agreement with the reported value for regular $R=1 / 4$ Gallager codes [44].

Notice that the reentrant nature of both the dynamical and critical boundaries, as we found solving the convolution (14), is confirmed by the decoding experiments. In the range of noise levels $0.1315<p<0.1665$, decoding is possible only in a range of finite temperatures, as indicated in the inset of Fig. 1. The boundary between decodeable and undecodeable regions reaches its maximal value at the Nishimori temperature; zero temperature decoding fails at the same noise level. The reentrant nature of the critical phase boundary increases below the Nishimori line, but eventually weakens as the temperature decreases further, a phenomenon that has already been discussed in a variety of different contexts $[45,46]$.
The fact that MPM and zero temperature BP-RS decoding do not coincide is not in contradiction with the results obtained in Ref. [47], which indicate that MAP and MPM decoding provide the same critical transition point. Zero temperature BP-RS decoding does not provide MAP results due to RSB effects. The latter and their effect on the phase diagram and on the reentrant nature of the phase boundaries will be further discussed in Sec. IV.

An independent calculation has been performed at zero temperature. In this case the recursion relations (13) simplify and one finds limiting expressions for the two functions $u\left(\left\{x_{i}\right\}, J\right), \quad a\left(\left\{x_{i}\right\}, J\right)$. Implementing numerically such asymptotic expressions would require an independent calculation where one explicitly changes the recursion relations (13) to their asymptotic $\beta \rightarrow \infty$ form. The importance of the polynomials $R(\sigma)$ becomes clear at this point. At any temperature considered, we distinguish the finite and zero temperature contributions, which corresponds to factorizing the largest exponent in the sum (11), so that the limit of zero temperature is particularly simple to handle in our approach and no explicit changes are required in the recursion relations (13). We observe that at zero temperature the local field distribution is not peaked at integer values as one might expect. We find that the relative weight of the background behind the integer-peaked distribution weakens as one consider the RSB solution, but restricting the distribution of the local fields to integer values, which results in a modification of the recursion relations (13) leads to different results. For LDPC (RS) decoding in the BSC this leads to an erroneous dynamical transition value of $p_{d} \simeq 0.121$, marked in Fig. 1 by a black filled dot, well below the RS zero temperature value of $p_{d}$ $\simeq 0.1315$ obtained by the full distribution. Similar behavior occurs in the binary erasure channel problem if one neglects the background distribution, as we will discuss in Sec. IV.

The initial condition $\pi^{0}(x)=(1-q) \delta(x-1)+q \delta(x+1)$, at $q=\frac{1}{2}$, has been used as the initial condition for the BSC analysis. A dependence of the solution of the convolution (15) on the initial condition for the local field has been observed. We studied the above convolution (15) for different choices of the initial condition in the form

$$
\pi(x)=(1-q) \delta(x-1)+q \delta(x+1) .
$$

The choice of $q=p$, where $p$ is the channel flip rate, results in the phase diagram of Fig. 3. Any choice of initial condition with $q>p$ (including the case $q=1 / 2$ considered above) leads to the dynamical transition line as shown in Fig. 1 (dashed line), except for the choice $q=p$ which leads to a different dynamical transition. The location of the critical transition remains unchanged.

For instance, using the initial condition $q=p$, the dynamical transition along the Nishimori line increases to $p_{d} \simeq 0.1734$, well above the known dynamical transition point. The physical interpretation of this behavior remains to be explained. Since it results from a simple change in the initial conditions of Eq. (12), one might argue that a properly modified decoding algorithm would enable one to decode above current $p_{d}$ values; this is a possibility that we cannot rule out and further research is needed in order to elucidate this point. 


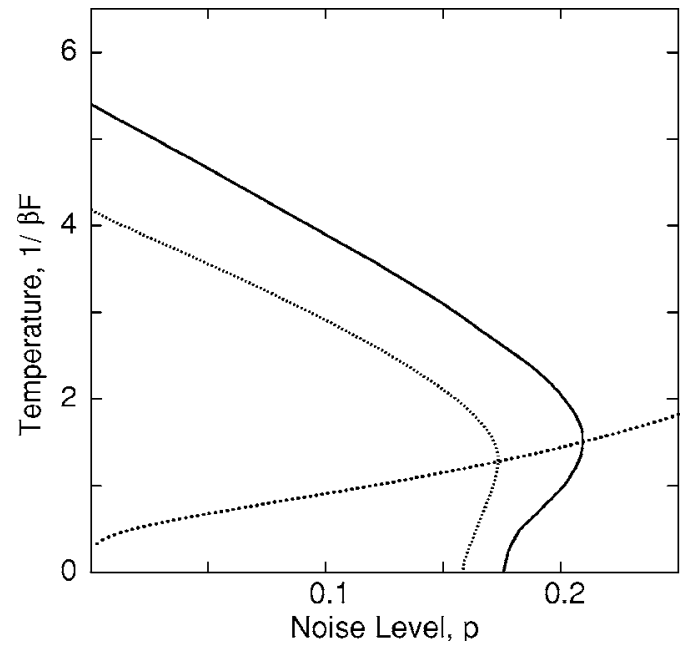

FIG. 3. The phase diagram of LDPC density evolution for the BSC with the initial condition (16). The location of the critical transition is unchanged unlike the location of the dynamical transition, which has been observed to increase. We observe, on the Nishimori line, a dynamical transition occurring at $p_{d} \simeq 0.1734$, well above the documented dynamical transition of the regular Gallager code of rate $R=1 / 4$.

We were able to perform a second set of decoding experiments, using the initial conditions that correspond to the choice $q=p$. The decoding results obtained coincide with the dynamical transition line in Fig. 3 but only for noise level values below the value $p_{d} \simeq 0.1665$. The reentrant nature of the dynamical transition line (the dynamical transition at zero temperature being $p_{d} \simeq 0.158$ ) is reduced in this case. Further study is required for understanding the significance of the choice of initial conditions on the decoding.

\section{Irregular Gallager codes}

For a constant $K$ value one can select noninteger connectivity values of average $\bar{C}$ and consider a modified form of the convolution (15) that involves a linear combination of terms corresponding to different connectivity values. Noninteger values of the multispin interaction parameter $K$ can also be easily accommodated in Eq. (15).

It has been reported that irregular low-density paritycheck codes outperform regular constructions [25,43].

We considered a generalized form of the convolution (15), (an explicit form is not provided for brevity) where both connectivity $C$ and multispin interaction $K$ take noninteger values such that the rate $R=1-\bar{C} / \bar{K}=1 / 4$ is preserved. In Fig. 4 we report the dynamical transition line for the regular construction $C=3, K=4(R=1 / 4)$ already considered in the previous section together with the dynamical transition lines for irregular constructions of two values $K=4$ and $K=3$ and $C=2$ and $C=3$, such that $\bar{C}=2.9,2.8,2.7$ (left to right) and $\bar{K} \simeq 3.87,3.74,3.6$. The dynamical transition value is observed to increase for these irregular constructions. This does not give a direct information on the specific pattern of irregularity that one should consider but provides quantitative information on the average connectivity and multispin interac-

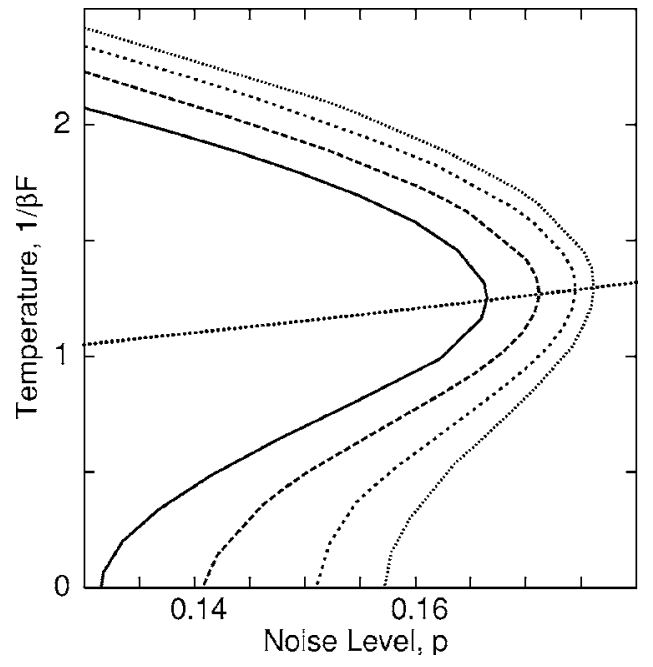

FIG. 4. Irregular LDPC phase diagram for the code rate $R$ $=1 / 4$. We show the dynamical transition for different values of the connectivity parameters $C$ and $K$. The solid line corresponds to the dynamical transition line of a regular construction with $C=3$ and $K=4$. The dashed, dotted, and thin-dotted lines correspond, respectively, to $\bar{C}=2.9,2.8,2.7$, at constant rate $R=1 / 4$. At lower average connectivity values, e.g., along the Nishimori line, the convolution (15) no longer flows to a ferromagnetic sink, but we observe a stable crossover to a distribution with large but finite average, so that successful decoding cannot be expected.

tion values one should investigate further. As for the regular case discussed earlier, a phase diagrammatic approach to the problem of irregular LDPC codes is important in order to obtain better understanding of the successful decoding properties of irregular LDPC codes. A similar behavior to the one shown in Fig. 3, but less pronounced, has also been observed for LDPC decoding with the initial conditions (16) when $q=p$.

\section{E. Finite connectivity problem with $C / K>1$}

We considered the finite-connectivity model (4) for the values of the connectivity $C=9$ and $K=4$. Despite the fact that it does not correspond to any Gallager LDPC code construction it is interesting to consider these connectivity parameters for two reasons: (i) They can represent code constructions in other variants of the LDPC family such as Sourlas or MN codes [25], and as such may also be linked to other LDPC-based applications. (ii) They give rise to a SG behavior which is of interest. In Fig. 5 we report the corresponding phase diagram obtained considering the convolution (15). An SG phase occurs in this case. At low values of the flip rate $p$ the convolution (12) flows to the ferromagnetic sink. In the vicinity of the dynamical transition line (marked in Fig. 5 as the solid black line) the trajectories under iteration of the above convolution cross over to an unstable distribution and then either flow to the ferromagnetic sink, below the dynamical transition, or to a stable nonferromagnetic distribution. For temperatures below the Nishimori line, at high enough values of the flip rate, the convolution instead flows to a spin-glass distribution. The initial condition used in this case was $q=1 / 2$. 


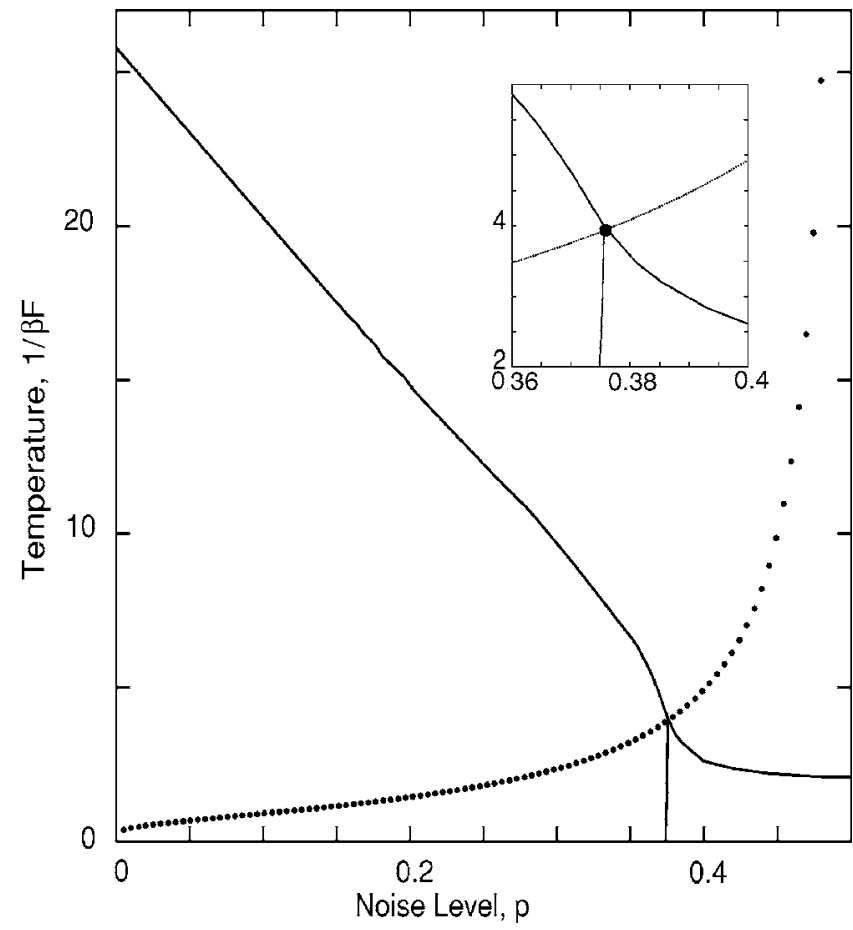

FIG. 5. The dynamical transition line for the finite-connectivity system with $C=9, K=4$. We report the location of the dynamical transition, shown in thick solid lines. At high enough values of the flip rate, the convolution (15) flows either to a stable, asymmetric local field distribution (at high enough temperatures), or to a symmetric SG distribution (below the Nishimori line). Before flowing to the corresponding sinks, the flow crosses over in the vicinity of either an unstable distribution above the Nishimori line, shown as the dotted line, or at the vicinity of a strong coupling unstable local field distribution below the Nishimori line. Similar behavior has been observed for the LDPC convolution (15) with the initial condition $q=1 / 2$. In that case the location of the dynamical transition occurs at lower values of the noise channel probability. The inset shows the location of the dynamical boundaries together with the Nishimori line.

\section{REPLICA SYMMETRY BREAKING}

The role of RSB for the Hamiltonian (4) is the subject of this and the following sections. The one-step RSB solution for the Bethe SG, as a special limiting case of the Hamiltonian (4), has been studied in detail long ago. In this case it is well known that the RS solution is incorrect [11]. Recently, the onset of RSB phenomena in some limiting cases of the problem (4) has been considered, relying on the frozen $\mathrm{SG}$ solution [9]. Consider the equation for the global order parameter $g\left(\left\{\sigma^{\alpha}\right\}\right)$, first introduced for the Bethe SG by Mottishaw [5] in the general case of a multispin interaction parameter $K$,

$$
\begin{aligned}
g\left(\left\{\sigma_{o}^{\alpha}\right\}\right)= & E_{J, \zeta}\left[\operatorname{Tr}_{\left\{\sigma_{i}^{\alpha} g^{C-1}\left(\left\{\sigma_{1}^{\alpha}\right\}\right) \cdots g^{C-1}\left(\left\{\sigma_{K-1}^{\alpha}\right\}\right)\right.}\right. \\
& \left.\times \exp \left(\beta J \sum_{\alpha} \sigma_{o}^{\alpha} \sigma_{1}^{\alpha} \cdots \sigma_{K-1}^{\alpha}+\beta F \sum_{j \alpha} \sigma_{j}^{\alpha}\right)\right],
\end{aligned}
$$

where $\alpha=1, \ldots, n$ labels different system replicas. The above expression holds in general, whether replica symmetry is broken or not (see Appendix Bfor the derivation). In the RS approximation one assumes the equivalence of replicas and the functional order parameter $g\left(\left\{\sigma^{\alpha}\right\}\right)$ depends only on the variable $\sigma=\Sigma_{\alpha} \sigma^{\alpha}$. In the limit of $n \rightarrow 0$, it can be shown to satisfy

$$
\begin{aligned}
g(\sigma)= & E_{J, \zeta} \int \prod_{j=1}^{K-1} \frac{d s_{j}}{2 \pi} g^{C-1}\left(i s_{j}\right) d u_{j} e^{i u_{j} s_{j}} \\
& \times \exp \left[-\frac{\sigma}{\beta} \tanh ^{-1}\left(\tanh (\beta J) \prod_{j=1}^{K-1} \tanh \left(\beta u_{j}-F \zeta\right)\right)\right] .
\end{aligned}
$$

This equation corresponds to the Bethe $\mathrm{SG}$ equation as soon as $K=2$ and for vanishing message prior $F=0$ [5]. A derivation of Eq. (18) is found in Appendix B, where we show the equivalence of the above expression with the set of recursion relations (12), for the local field and conjugate distributions. In the one-step RSB approximation one assumes instead that the functional order parameter $g\left(\left\{\sigma^{\alpha}\right\}\right)$ depends only on the group variables $\sigma_{M}=\Sigma_{\gamma} \sigma_{M}$, where $\alpha=(M, \gamma)$ is the replica index, $M=1, \ldots, n / m$ and $\gamma=1, \ldots, m$. In this case one finds that the corresponding equation for the functional order parameter $g\left(\left\{\sigma^{\alpha}\right\}\right)$ becomes

$$
\begin{aligned}
& g\left(\left\{\sigma_{M}\right\}\right)=\mathcal{N}^{-1} E_{J, \zeta} \int \prod_{j=1}^{K-1} \prod_{M=1}^{n / m} \frac{d s_{M j}}{2 \pi} g^{C-1}\left(i s_{M j}\right) \\
& \int \prod_{M j} d r_{M j} e^{i r_{M j} s_{M j} \exp \left(-\sum_{M} \sigma_{M} u\left(\left\{r_{M j}\right\}, J\right)\right)} \\
& \quad \times \exp \left(\frac{m}{2} \sum_{M} a\left(\left\{r_{M j}\right\}, J\right)\right),
\end{aligned}
$$

where the normalization is given by

$$
\begin{gathered}
\mathcal{N}=\int \prod_{j=1}^{K-1} \prod_{M=1}^{n / m} \frac{d s_{M j}}{2 \pi} g^{C-1}\left(i s_{M j}\right) \\
\int \prod_{M j} d r_{M j} e^{i r_{M j} j_{M j}} \prod_{j} \cosh ^{m}\left(\beta r_{M j}\right) .
\end{gathered}
$$

The corresponding one-step RSB convolution follows, assuming that the order parameter factorizes according to

$$
g\left(\left\{\sigma_{M}\right\}\right)=\prod_{M=1}^{n / m} f\left(\sigma_{M}\right) .
$$

The assumptions behind the factorized Ansatz (20), where the local field distribution is independent of the intragroup index, are discussed in Ref. [10]. In this case the one-step RSB convolution (19) becomes

$$
\pi(x)=E_{\zeta} \int \prod_{i=1}^{C-1} d \hat{x}_{i} \hat{\pi}(\hat{x}) \delta\left(x-\sum_{i=1}^{C-1} \hat{x}_{i}-F \zeta\right),
$$




$$
\begin{aligned}
\hat{\pi}(\hat{x})= & \mathcal{N}^{-1} E_{J} \int \prod_{j=1}^{K-1} d x_{j} \pi\left(x_{j}\right) \exp \left[\mu a\left(\left\{x_{j}\right\}, J\right)\right] \\
& \times \delta\left(\hat{x}-u\left(\left\{x_{j}\right\}, J\right)\right),
\end{aligned}
$$

where the normalization $\mathcal{N}$ is given by

$$
\mathcal{N}=\int \prod_{j=1}^{K-1} d x_{j} \pi\left(x_{j}\right) \exp \left[\mu a\left(\left\{x_{j}\right\}, J\right)\right],
$$

and where $\mu=m / \beta$. The above equations are valid, under the assumption (20), at finite temperatures and arbitrary connectivity values $C$ and $K$. After its very first formulation [10] the convolution (21) has been studied in a range of different contexts, from random $K$-SAT to the coloring problem [49]. Using the identity

$$
\exp \left[\mu a\left(\left\{x_{j}\right\}, J\right)\right]=\left[\frac{2 \cosh \left[u\left(\left\{x_{j}\right\}, J\right)\right]}{\prod_{j} \cosh \left(\beta x_{j}\right)}\right]^{\mu},
$$

and substituting it in Eq. (21) one recovers the one-step RSB equations that have been also derived using the factorized variational approach $[7,10,31,48]$. Notice that the factorized Ansatz of Refs. $[7,10]$ and those obtained by variational approaches elsewhere are equivalent, in spite of some degree of arbitrariness [50] in the way one redistributes the weighting between the two equations (21). We prefer the notation that involves the exponential of $a\left(\left\{x_{j}\right\}\right)$, rather than the less obvious (and numerically more difficult to implement) parallel expression that follows from the right hand side of Eq. (23) $[48,49]$. The simplified expression of the free energy presented in Sec. III was obtained by virtue of the same identity (23), and the degree of freedom in writing the free energy was already discussed for the Bethe SG [the right hand side of Eq. (23) becoming, for $K=2$, the function $c(x, J)[11]]$.

\section{A. Bethe SG}

We apply the method described in Sec. III to study the one-step RSB convolution (21) of the Bethe SG, which has been extensively studied previously and recently considered also in its ferromagnetically biased version [15]. In this case the distribution of the quenched random variable $J$ is as in Eq. (3), where the parameter $\rho \in[0,1]$ ranges between $\rho=1$ for a ferromagnet and $\rho=0$ for the unbiased Bethe SG. The Bethe SG corresponds indeed to the original problem (1) at $K=2$. We will present results obtained at zero temperature; in this limit we find the following expression for the free energy $f(\mu)$ :

$$
f(\mu)=\Delta E^{(1)}-\frac{C}{2} \Delta E^{(2)},
$$

where explicit expressions for the site and link contributions to the ground state energy become

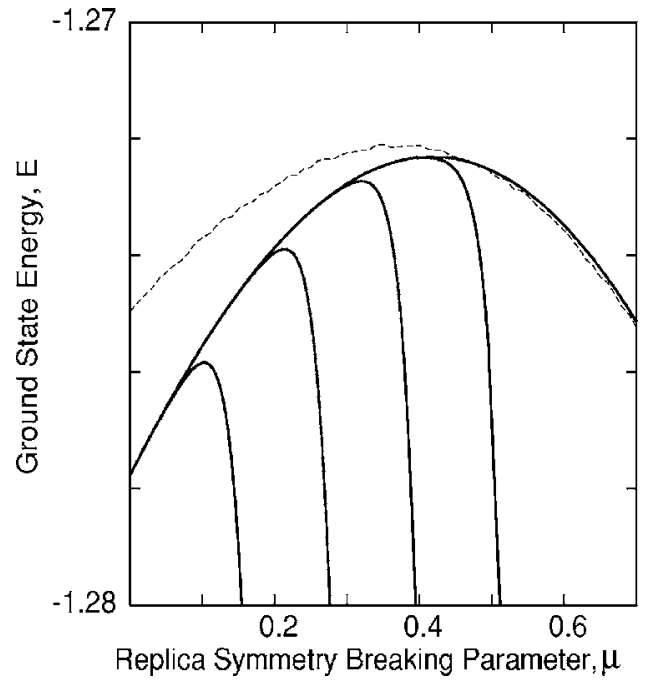

FIG. 6. The ground state energy as a function of the replica symmetry breaking parameter for different bias values (from left to right) $\rho=0.66,0.68,0.70,0.72,0.74$. We also show in the thin dotted line the ground state energy including the background distribution as in Ref. [3].

$$
\begin{aligned}
\exp \left(-\mu \Delta E^{(1)}\right)= & \int \prod_{j=1}^{C} d x_{j} \pi\left(x_{j}\right) \\
& \times \exp \left(\mu \sum_{j=1}^{C} a\left(\left\{x_{j}\right\}, J_{j}\right)+\mu\left|\sum_{j=1}^{C} u\left(\left\{x_{j}\right\}, J_{j}\right)\right|\right) \\
\exp \left(-\mu \Delta E^{(2)}\right)= & \int \prod_{j=1}^{2} d x_{j} \pi\left(x_{j}\right) \\
& \times \exp \left(\mu \max _{\sigma_{1}, \sigma_{2}}\left(x_{1} \sigma_{1}+x_{2} \sigma_{2}+J \sigma_{1} \sigma_{2}\right)\right),
\end{aligned}
$$

corresponding, at $\mu=0$ and $K=2$, to the RS equation (8) in the zero temperature limit and neglecting the prior term in Eq. (4). Similar expressions have been discussed in Ref. [11]. In the limit of zero temperature one finds $a(J, x)=|x|+\delta_{x, 0}$ and $u(x, J)=J S(x)$, being $S(x=0)=0$, and $S(x \neq 0)=\operatorname{sgn}(x)$. In what follows we present results obtained under the integer peaked Ansatz as well as for the full distribution. The relative importance of noninteger values in the distribution that solves the above convolution (21) is rather limited in this case. The background behind the integer peaked values weakens as one considers the one-step RSB case, and may disappear in a fully replica symmetry broken scenario [11]. In Fig. 6 we show the ground state energy as a function of the replica symmetry breaking parameter $\mu$ for different values of the ferromagnetic bias using both the integer peaked Ansatz and the full local field distribution. The ground state energy one extrapolates including the background calculation is higher than the one obtained within the three peak Ansatz [10]. In Fig. 7 we show the corresponding complexity or configurational entropy, that can be obtained as a parametric plot in terms of the RSB parameter $\mu$, the free energy $f(\mu)$ 


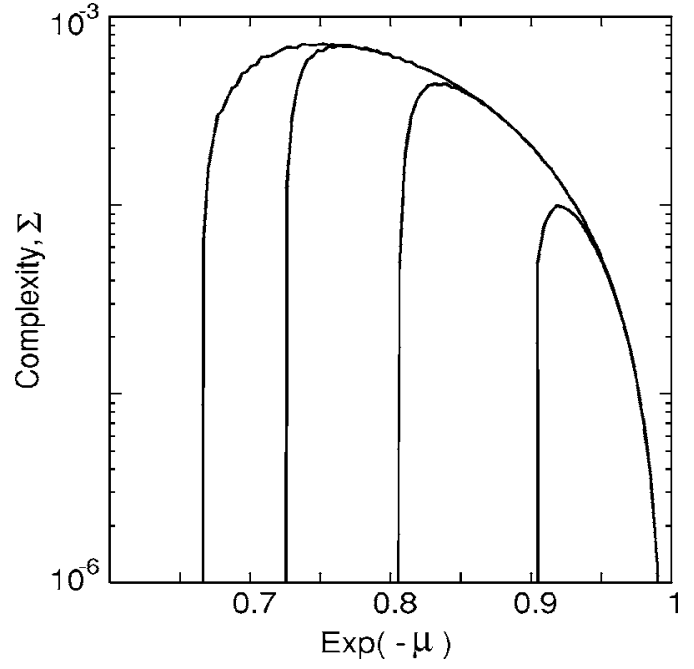

FIG. 7. The complexity $\Sigma$ as a function of $\exp (-\mu)$ for different ferromagnetic bias values $\rho=0.66,0.68,0.70,0.72,0.74$, within the three peak Ansatz.

being its Legendre transform [4]. We observe a positive complexity for all reported values of $\rho$. At higher values of the ferromagnetic bias $\rho>\rho^{*} \simeq 0.74$ a positive complexity has not been observed. One should also consider that the above values should be extrapolated in the limit of a large number of bins, so that one expects a slightly higher value $\rho^{*} \simeq 0.75$. In Fig. 8 we show the magnetization in the replica symmetric and one-step replica symmetry broken solution at different values of the ferromagnetic bias. At values $\rho<0.65$ one recovers the known results for the unbiased Bethe SG. The solid line indicates the magnetization computed in the replica symmetric approximation (notice that

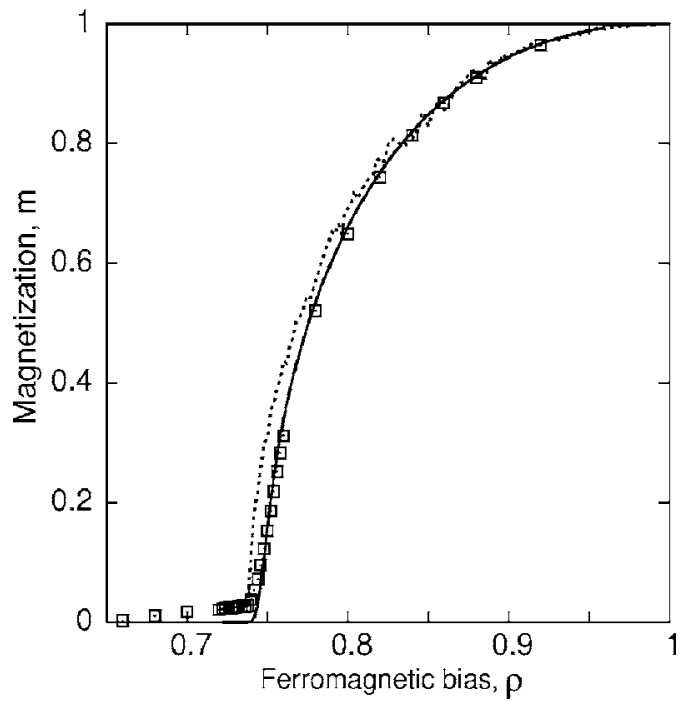

FIG. 8. The magnetization as a function of the ferromagnetic bias $\rho$. The thick solid line corresponds to the RS calculation obtained with $4096 \times 4$ bins. Square boxes correspond to the one step RSB calculation if one neglects the background local field distribution. Finally the dotted line represents the one step RSB calculation including the background calculation, according to Eq. (21), obtained with $32768 \times 4$ bins. this is a computed magnetization for a finite number of bins, so that it approaches the expected value of $\rho_{R S}=0.75$ from below), while the square boxes represent the magnetization in the one step RSB approximation without considering the background. For each value of $\rho$, we extrapolated the magnetization that corresponds to the value of $\mu$ where the ground state energy is maximized. Similarly, the dashed line points to the results obtained considering the true convolution (12), that includes noninteger values of the local field. The putative size of the so called mixed phase [15], if any, is rather small and does not agree with previous estimates (we observe the magnetization within the RSB calculation to vanish at values of $\rho \simeq 0.75$, if one considers a large enough number of bins within the calculation, implying that the size of the mixed phase, if any, is vanishingly small). It should be said, however, that the calculation of Ref. [15] considered a full one step RSB calculation, in the sense of Ref. [11], where the factorized approximation is relaxed. To investigate this point better, we perform the following: instead of implementing the convolution (12) within the factorized approximation $[7,10]$, we choose a population of $5 \times 10^{5}$ distributions (including a site dependence). At each step of the iteration process we consider pairwise convolutions of randomly chosen local field distributions corresponding to different sites; after performing a number of iteration steps of the order of the population size, which correspond to a transient regime, we obtain the equilibrium population. According to our calculation, the local field distributions become site independent, meaning that the factorized approximation provides similar results to those of the full RSB Ansatz. If one compares Fig. 7 with the corresponding plot in Ref. [15], where a similar calculation of the complexity is presented, we see that the location of the maximum of the complexity as a function of $\mu$ decreases for increasing $\rho$ values (see Fig. 7 ), as one might intuitively expect, increasing the ferromagnetic bias.

\section{B. Binary erasure channel}

In the case of the BEC discussed in Sec. II, the form of the convolution (15) simplifies and the distribution of the local field, and its conjugated distribution become bimodal. Despite its simplicity, the binary erasure channel problem is of practical relevance [51,52]. For the BEC one writes the convolution (15) as

$$
\begin{aligned}
& \pi(x)=\rho \delta(x)+(1-\rho) \delta_{\infty}(x), \\
& \hat{\pi}(\hat{x})=\hat{\rho} \delta(\hat{x})+(1-\hat{\rho}) \delta_{\infty}(\hat{x}),
\end{aligned}
$$

where $\delta_{\infty}(\cdot)$ is a delta function located at $+\infty, \rho(x)$ is the distribution of the effective local field, and $\hat{\rho}(\hat{x})$ its conjugate distribution. In the one-step RSB case, the resulting convolution [31] involving the two distributions $\rho(x)$ and $\hat{\rho}(\hat{x})$ is given by

$$
\rho(x)=\int \prod_{l=1}^{C-1} d \hat{x} \hat{\rho}(\hat{x}) \delta\left(x-\sum_{l=1}^{C-1} \hat{x}_{l}\right)
$$




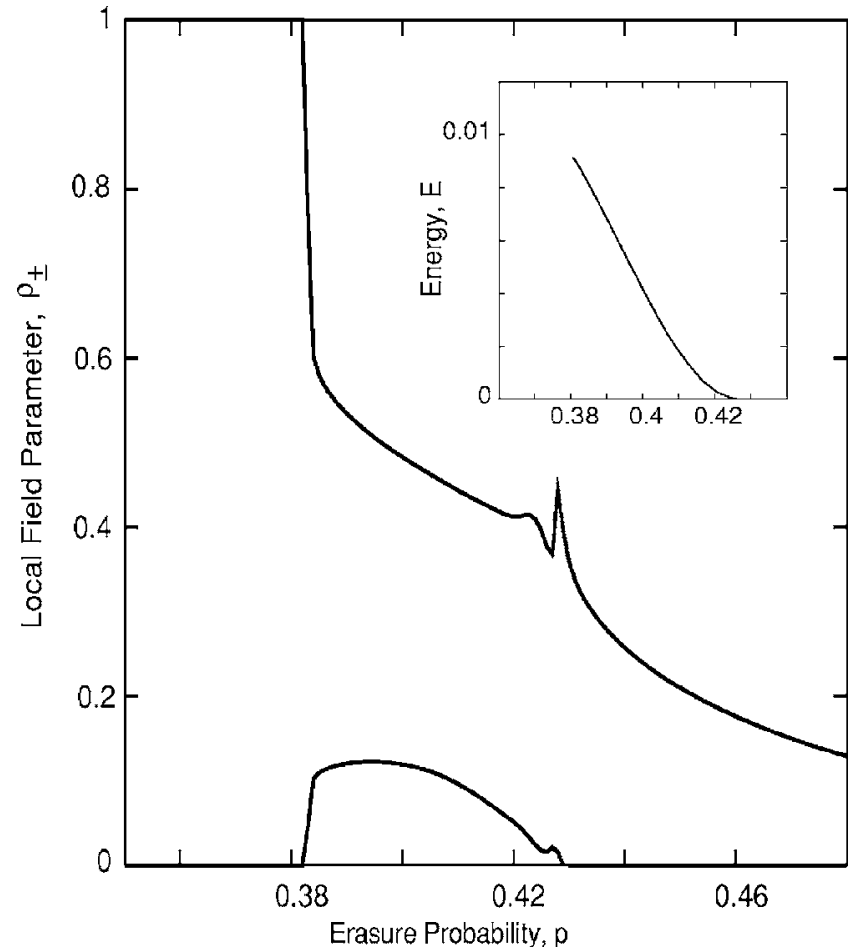

FIG. 9. For the code rate $R=1 / 2$ and a BEC channel we report the local field parameter $\rho_{ \pm}$as a function of the erasure probability $p$, at zero temperature, within the integer value Ansatz, (29). The continuous lines represent the probability of a negative $\rho_{-}$(lower) and positive $\rho_{+}$(higher) local field, respectively. The inset shows the computed energy as a function of the erasure probability.

$$
\begin{aligned}
\hat{\rho}(\hat{x})= & \sum_{\nu}^{K-1} f_{\nu} \mathcal{N}_{\nu} \int \prod_{i=1}^{\nu} d x_{i} \rho\left(x_{i}\right) \exp \left[\mu a\left(\left\{x_{i}\right\}, J\right)\right] \\
& \times \delta\left(\hat{x}-u\left(\left\{x_{i}\right\}, J\right)\right),
\end{aligned}
$$

where $f_{\nu}=\left(\begin{array}{l}K-1 \\ \nu\end{array}\right)$ are the binomial coefficients and $\mathcal{N}_{\nu}$ the set of normalization coefficients defined by

$$
\mathcal{N}_{\nu}=\int \prod_{i=1}^{\nu} d x_{i} \rho\left(x_{i}\right) \exp \left[\mu a\left(\left\{x_{i}\right\}, J\right)\right] .
$$

One can readily check that the above convolution reduces to the factorized approximation [31] using identity (23). We note again that this factorized Ansatz, as already discussed for the case of the BSC, coincides with the Ansatz (20) of Refs. $[7,10]$. We consider an explicit expression for the one step RSB free energy that involves the binomial coefficients of order $K$. Within the factorized hypothesis [7,10], the expression for the free energy is easily computed as a function of the RSB parameter $\mu$. We present results for the BEC at zero temperature for values of the code rate $R=1 / 4$ and $R=1 / 2$ (note that we also consider in what follows the integer peak Ansatz for the local field distribution [31] in order to compare with the more general case of noninteger local fields). In the inset of Fig. 9 we show the energy as a function of the erasure probability $p$ for the rate value $R=1 / 2$ within the integer valued local field approximation. Notice that the integer value approximation corresponds to having

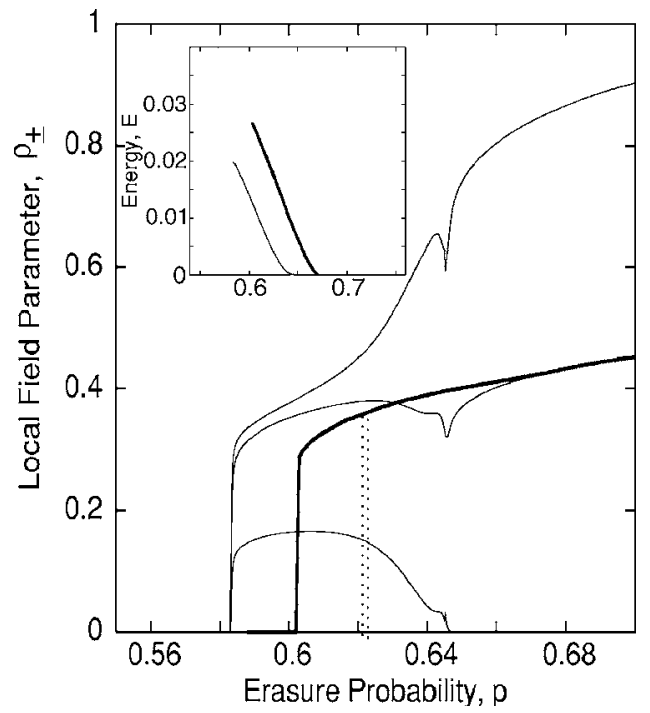

FIG. 10. For a code rate $R=1 / 4$ and a BEC channel at zero temperature, we report the local field parameters as a function of the erasure probability $p$, for the Ansatz (29) denoted by thin lines, corresponding to $\rho_{-}, \rho_{-}+\rho_{0} / 2$, and $\rho_{0}$. We then report (thick line) the results for the local field probability according to the full convolution (27), as it is in the simplest RS approximation $\mu=0$, corresponding to $\rho_{x<0}$, where $\rho_{x>0}=1-\rho_{x<0}$. The dashed vertical line corresponds instead to the location of the dynamical transition within the one step RSB calculation. The inset shows the computed energy as a function of the erasure probability within the three peak Ansatz (thin line) and in the case of the full convolution of the local field being considered (thick solid line).

only three bins in our approach of Sec. III. In Fig. 10 we compare the results within and without the integer approximation for a code rate $R=1 / 4$. The dynamical transition is defined as the point where suboptimal solutions emerge with the corresponding complexity being nonzero, as already discussed for the BSC channel (see Fig. 10). The location of the dynamical transition is also the point where the recursion (21) flows to a subdominant distribution, rather than the ferromagnetic sink. This coincides with the point where algorithms devised for real decoding experiments fail to decode the original message. Within the RS framework, we find that the location of the dynamical transition changes significantly if noninteger values of the local field are taken into account.

In the three peak Ansatz the local field distribution is simply defined as [21]

$$
\rho(x)=\rho_{+} \delta(x-1)+\rho_{-} \delta(x+1)+\rho_{0} \delta(x) .
$$

The calculation we performed within the integer local field approximation (for rates $R=1 / 2$ and $R=1 / 4$ in Figs. 9 and 10 , respectively) might suggest to interpret the point $p^{*} \simeq 0.4294$ for the rate value $R=1 / 2$ as the dynamical transition point [31]. If, however, one compares this (see Fig. 10) with the results of the local field distribution that now includes noninteger values (the thick solid line, corresponding to $\rho_{x>0}$ ) we see that the location of the dynamical transition is rather different. Moreover, the behavior of the local field probability $\rho_{ \pm}$around the value $p^{*} \simeq 0.4294$, within the three peak Ansatz is rather suspicious. Indeed, including noninte- 


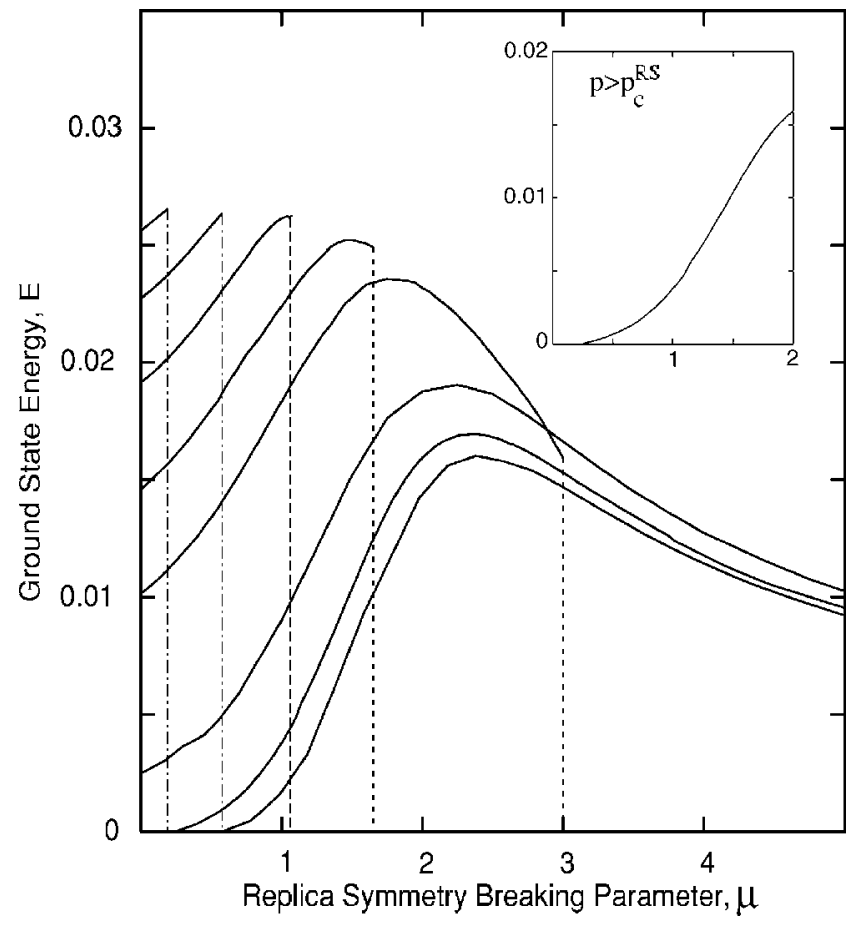

FIG. 11. The one step RSB free energy as a function of the parameter $\mu$ for different values (left to right) of the erasure probability $p=0.606,0.613, p=0.62,0.63,0.64,0.66$, and $p=0.67$. The first two curves, corresponding to y $p=0.606, p=0.613$ drop to zero before a maximum is reached as a function of $\mu$, indicating the absence of the physical branch of the complexity. In contrast, around values of $p \simeq 0.625$ the energy reaches a maximum before dropping to zero, so that the physical branch of the complexity appears. The inset shows the ground state energy as a function of the RSB parameter $\mu$, at a flip rate above the critical transition point.

ger values of the local field turns out to be crucial for having a quantitative description of the dynamical transition and possibly explains the disagreement with numerical experiments that has been reported lately for the $R=1 / 2$ BEC problem [31]. If one avoids the three peak Ansatz, the nature of the flows dictated by the recursion (27) is smooth as it always leads to a well-behaved behavior of the probability densities $\rho_{x>0}$ and $\rho_{x<0}$. Up to this point all considerations are still within the RS approximation.

Moreover, considering the full convolution (27) as we do in what follows, the location of the dynamical transition in the one-step RSB solution becomes higher than that obtained within RS, which arguably implies that one may be able to construct the algorithmic analog of the RSB recursion (27) to improve decoding performances. Similarly, within the RSB calculation, the location of the critical transition is increased for the rate $R=1 / 4$ considered above and found to be very close to the Shannon bound.

We iterated the convolution (27) within the one-step RSB approximation described in Sec. VI and computed the free energy as a function of the RSB parameter $\mu$ for several values of the erasure probability $p$, above the dynamical transition point $p_{d} \simeq 0.602$ as reported in Fig. 11. In this range of $p$ values, the free energy grows as a function of $\mu$, dropping

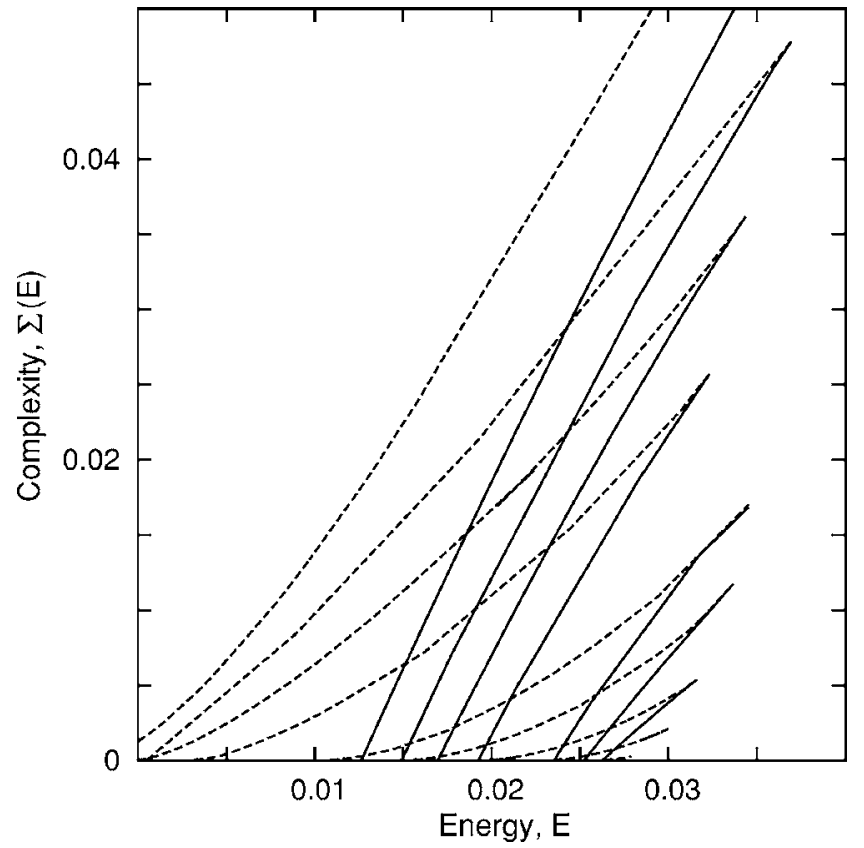

FIG. 12. Complexity $\Sigma(E)$ as a function of the ground state energy $E$ for different values of the erasure probability $p$. From right to left $p=0.606,0.613,0.62,0.63,0.64,0.66,0.67,0.68$, and $p=0.69$ for the code rate problem $R=1 / 4$.

to the ferromagnetic free-energy value (being $f_{\text {Ferro }}=0$ for the BEC case) at some critical $\mu^{*}$. At higher values of the erasure probability $p$, the free energy eventually drops to zero after having reached its maximum (see Fig. 11). According to the standard recipe of maximizing the free energy with respect to $\mu$ [34], we extrapolate the value of the dynamical transition within the one-step RSB calculation, finding the value of $p_{d}^{R S B} \simeq 0.621$, indicated in Fig. 10 by the dotted line, well above the $\mathrm{RS}$ value $p_{d}^{R S} \simeq 0.602$. The corresponding complexity is shown in Fig. 12, for different values of the erasure probability considered. We observe two branches, a physical (right) and an unphysical one (left) as explained in Ref. [11]. From the former branch we extrapolated the RSB energy value that corresponds to the noise channel probability. Notice that above the RS dynamical transition we do observe, for values of $p \simeq 0.606,0.613,0.62$, that the second, physically relevant, branch is not present. This can be explained by the corresponding curves for the free energy (Fig. 11) that do not reach a maximum and drop instead to the ferromagnetic energy value $(f=0)$. The dynamical transition within the RSB calculation increases.

If one considers the free energy as a function of the RSB parameter $\mu$ at values of the erasure probability slightly higher than that of the RS critical transition point, one finds that the maximum is located at positive energies, implying that the critical transition point also changes under the one step RSB calculation. In this way (Fig. 13) we extrapolated the value of the critical transition, within the RSB approximation, to $p_{c}^{R S B} \simeq 0.7450 \pm 0.05$, in excellent agreement with rigorous bounds that have been reported recently [53] and those obtained from approximate entropic calculations [22]. Note that Shannon's bound for the specific code rate considered is $1-R=3 / 4$. In comparison, the critical $\mathrm{RS}$ transition 


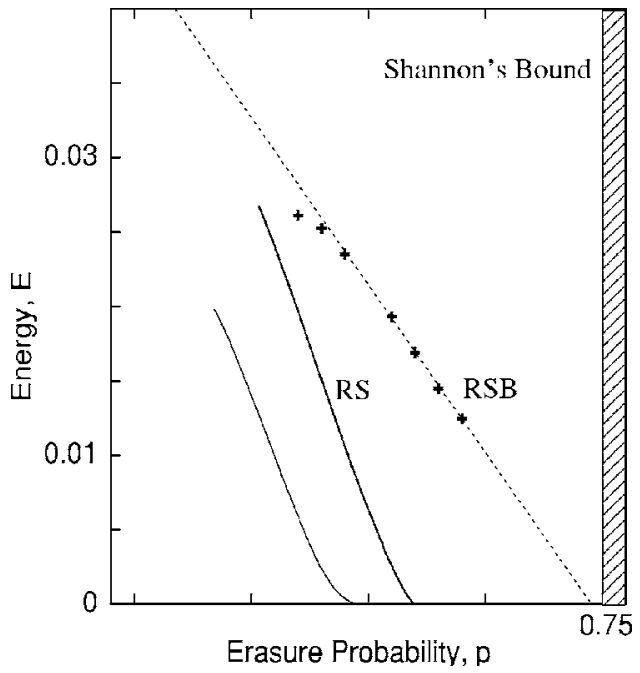

FIG. 13. The RS and RSB energy as a function of the erasure probability. The Shannon bound corresponding to the rate $R=1 / 4$ being $1-R$. The points (bold crosses) are extrapolated from the physical branch of the complexity at $\Sigma=0$ and corresponds to the maximum of the free energy as a function of the RSB parameter $\mu$. The thin line corresponds to the factorized Ansatz.

point is $p_{c}^{R S} \simeq 0.6695$. The fact that the dynamical and critical transition points increase within the one step RSB calculation might be surprising at first. In the next section we will show that a similar situation is observed for the BSC at temperatures below the Nishimori condition [28,54]. It is also reasonable to believe that the above one step RSB calculation is exact for this problem $[11,49]$. The above findings concerning the location of the critical (RSB) transition seem to support this belief.

\section{Binary symmetric channel}

In this section we return to the BSC (4) and employ the same considerations as discussed for the BEC in the previous section. We consider the convolution (21) along the Nishimori line $\beta=1$. The location of the dynamical transition has been discussed in Sec. III where we found the value of $p_{d}^{R S}$ $=0.1665$ within the RS approximation, in agreement with probabilistic decoding estimates. Not surprisingly [54], if one looks at the free energy as a function of the RSB parameter $\mu$ for values of the BSC noise probability $p>p_{d}$, one finds that the free energy decreases as a function of $\mu$ (see the inset in Fig. 14), indicating that the RS solution is dominant, although at higher values of the replica symmetry breaking parameter the free energy increases again and finally drops to the ferromagnetic free-energy value, indicating that, within the one step RSB solution, it is possible to recover the ferromagnetic state at some value of the RSB parameter, below the critical transition $p_{c}$. At temperatures well below the Nishimori line, e.g., $\beta=4.0$, we observe the following behavior: above the RS dynamical transition $p_{d}^{R S} \simeq 0.146$ the free energy increases as a function of the RSB parameter $\mu$, dropping to the corresponding ferromagnetic free energy at some value $\mu^{*}$, before reaching a maximum, as discussed above. This means that the second, physi-

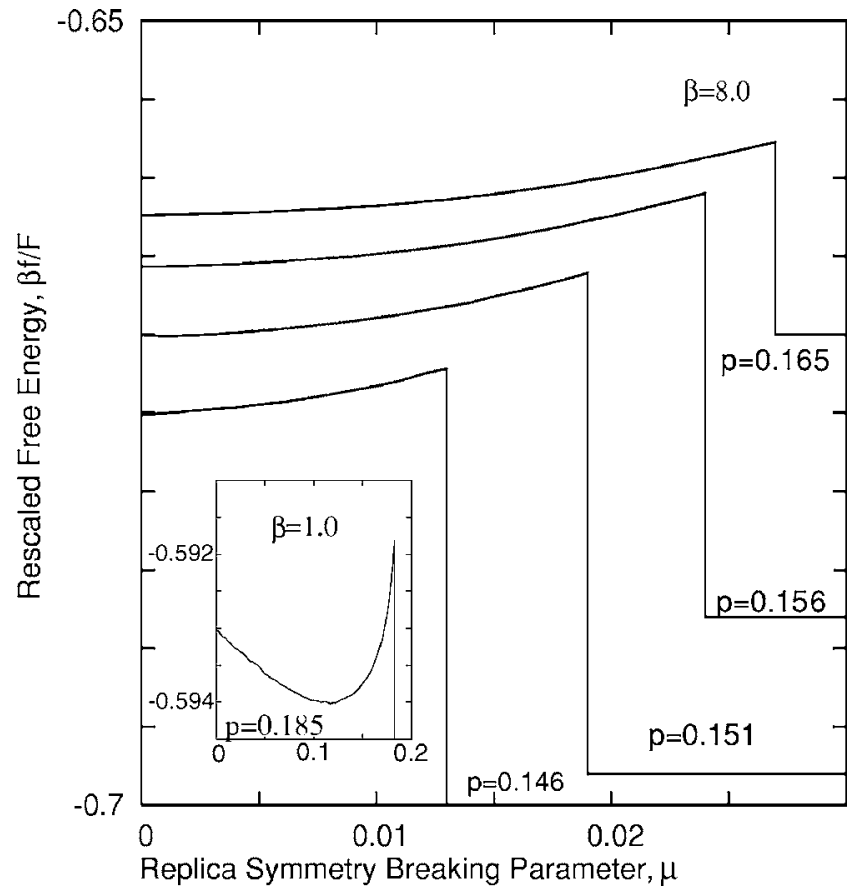

FIG. 14. The Gallager free energy as a function of the RSB parameter $\mu$ for different values of the BSC noise $p$ and temperature (the inset shows the free energy at the Nishimori temperature for a flip rate $p=0.175$, above the RS dynamical transition point).

cal branch of the complexity is not present. At higher values of the flip rate, i.e., $p \simeq 0.19$ the second branch of the complexity (see Fig. 15) appears, indicating a nonzero complexity, i.e., of nonferromagnetic solutions, and the location of

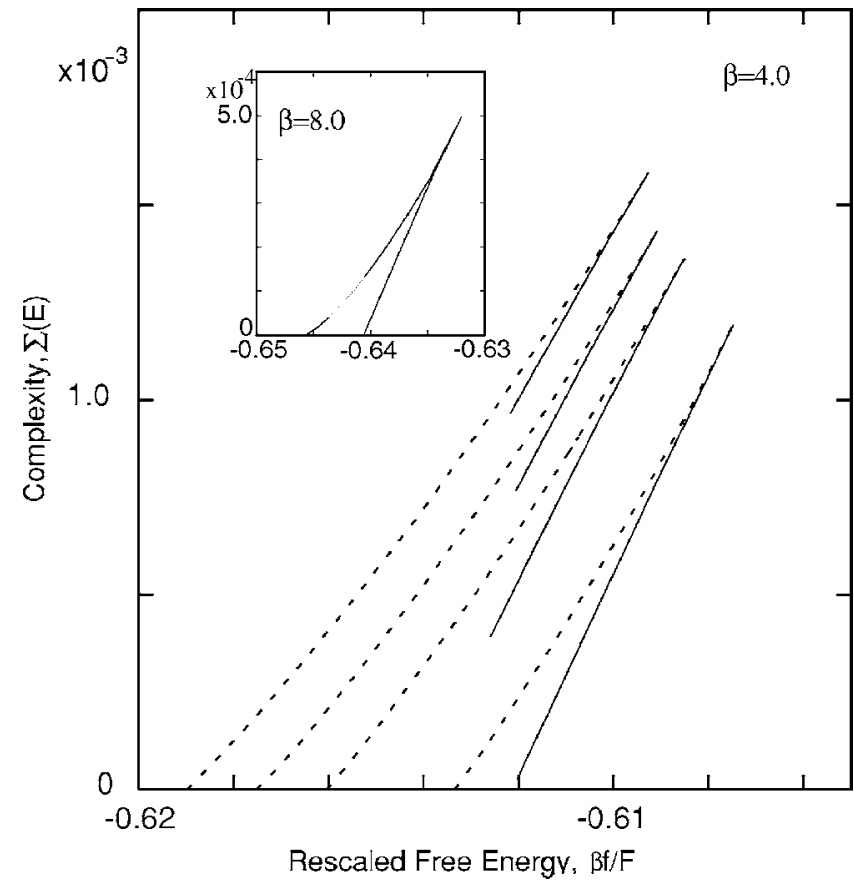

FIG. 15. The Gallager complexity for a BSC noise level of $p=0.195,0.1975,0.20,0.205$, at temperature $\beta=4.0$, below the Nishimori line. The inset shows the complexity at the temperature value $\beta=8.0$ and noise level $p=0.185$. 
the dynamical transition. The corresponding complexity for LDPC decoding at $p \simeq 0.195,0.1975,0.20,0.205$ is reported in Fig. 15 at temperature values $T=0.25$. These findings imply that the location of the dynamical transition under RSB is higher than the RS value. The location of the critical transition point is determined by the lowest flip rate where an equality between the ferromagnetic free energy and that of the emerging nonferromagnetic solutions is observed. In the numerical studies presented here we find that this almost coincides with the emergence of suboptimal solutions at temperatures below the Nishimori condition. It is also important to mention that within the one step RSB calculation we present here, the critical transition line $p_{c}$ still presents a reentrant behavior as discussed for the RS case. This is shown by comparing results obtained at the Nishimori condition and $p=0.185$, shown in the inset of Fig. 14, with those reported for $T=0.25$ and $T=0.125$ in Fig. 15. The reason for the discrepancy between our numerical results and the general belief that MAP and MPM decoding should exhibit the same critical value $[47,55]$ is unclear.

The inset of Fig. 14 shows the computed free energy as a function of the RSB parameter $\mu$ at the Nishimori temperature channel noise $p=0.174$. We conclude by pointing out that below the Nishimori line, the reentrant nature of the dynamical transition is still observed within the one step RSB calculation.

\section{CONCLUSIONS AND FUTURE PERSPECTIVES}

We introduced a different method to compute the phase diagram of finite connectivity SG model systems and discussed the connection with LDPC codes. We extended the one step RSB theory, originally formulated for the Bethe SG problem, to the Gallager LDPC decoding for two different type of corruption processes, BEC and BSC. For the BEC at zero temperature we found that the location of the dynamical transition increases with respect to the RS case. Our estimate for the RSB critical transition value is a mere one percent away from capacity, differently from the RS estimate and in excellent agreement with results reported elsewhere [53]. The critical and dynamical properties of LDPC decoding have also been studied for the case of the BSC. The reentrant nature of the RS LDPC phase diagram and the impact of RSB in the low-temperature regime has also been studied. We found that below the Nishimori line the noise level that corresponds to the dynamical transition increases within the RSB theory. Possible implications to improve the decoding performances of the state-of-the-art error correcting codes are one of the perspectives we wish to address. We would like to construct the algorithmic analog of the convolution (21) in order to improve the decoding performances as predicted by the RSB theory.

The method used to determine the phase diagrams in this work is very promising. It allows one to study, without loss of continuity, rather different problems, ranging from the $d$-dimensional hierarchical SG to finite-connectivity meanfield models. In this sense it would be very interesting to apply this method to clarify a few open issues. On the one hand we would like to investigate, within the one step RSB theory, the finite temperature phase diagram of the Bethe SG in a magnetic field. On the other hand, it would be interesting to explore the presence, if any, of RSB phenomena in $d$-dimensional SG hierarchical model systems.

\section{ACKNOWLEDGMENTS}

This work was supported in part by the European Community's Human Potential Programme under Contract No. HPRN-CT-2002-00319 and from EVERGROW, IP No. 1935 in FET, EU FP6.

\section{APPENDIX A: EQUIVALENCE OF THE RS EQUATIONS}

Considering the replicated partition function

$$
\left\langle\mathcal{Z}^{n}\right\rangle_{\mathcal{A}, \zeta, J}=E_{\mathcal{A}, J, \zeta} \operatorname{Tr}_{\left\{S_{j}^{\alpha}\right\}} e^{\beta F \sum_{\alpha, k} \zeta_{k} S_{k}^{\alpha}+\beta \sum_{\alpha, k} \mathcal{A}_{\mu} J} S_{\mu}^{\alpha} S_{i_{1}}^{\alpha} \cdots S_{i_{K}}^{\alpha}
$$

one can perform the average over the corresponding underlying geometry in Eq. (A1) introducing an integral representation for the finite-connectivity constrain $\delta\left(\Sigma_{\mu l i} \mathcal{A}_{\mu}-C\right)$ in the spirit of Ref. [3], defining the set of overlaps $q_{\alpha_{1} \cdots \alpha_{m}}$ and conjugated functional order parameter $\hat{q}_{\alpha_{1} \cdots \alpha_{m}},[20]$. This leads, under the RS Ansatz,

$$
\begin{aligned}
& q_{\alpha_{1} \cdots \alpha_{m}}=\int d x \pi(x) x^{m}, \\
& \hat{q}_{\alpha_{1} \cdots \alpha_{m}}=\int d \hat{x} \hat{\pi}(\hat{x}) \hat{x}^{m},
\end{aligned}
$$

to the following expression for the free energy:

$$
\begin{aligned}
\beta f= & -\frac{C}{K} \ln \cosh \beta J-\frac{C}{K} E_{J} \int\left(\prod_{l=1}^{K} d x_{l} \pi\left(x_{l}\right)\right) \\
& \times \ln \left(1+\tanh (\beta J) \prod_{j=1}^{K} \tanh \left(\beta x_{j}\right)\right) \\
& +C \int d x d \hat{x} \pi(x) \hat{\pi}(\hat{x}) \ln [1+\tanh (\beta x) \tanh (\beta \hat{x})] \\
& +C \int d y \hat{\pi}(\hat{x}) \ln \cosh (\beta \hat{x})-E_{\zeta} \int \prod_{l=1}^{C} d y_{l} \hat{\pi}\left(x_{l}\right) \\
& \times\left[2 \cosh \beta\left(\sum_{l=1}^{C} \hat{x}_{l}+F \zeta\right)\right] .
\end{aligned}
$$

The corresponding saddle point equations (14) are derived differentiating the above expression with respect to the local field $\pi(x)$ and its conjugated distribution $\hat{\pi}(\hat{x})$. In order to obtain the free energy (8), the second and third term in Eq. (A3) can be combined via Eq. (14) to obtain

$$
\begin{aligned}
I I+I I I \equiv & +C \frac{K-1}{K} \Delta f^{(2)}-C \frac{K-1}{K} \ln \cosh \beta J-C(K-1) \\
& \times \int d x \pi(x) \ln \cosh (\beta x) .
\end{aligned}
$$

The fourth term can be written as 


$$
\begin{aligned}
I V \equiv & \Delta f_{b}^{(1)}+C \ln \cosh (\beta J)+C(K-1) \\
& \times \int d x \pi(x) \ln \cosh (\beta x),
\end{aligned}
$$

where we wrote $\Delta f^{(1)}=\Delta f_{a}^{(1)}+\Delta f_{b}^{(1)}$ and where the last term in the free-energy expression above is nothing but $\Delta f_{a}^{(1)}$. It is easy to see that several terms cancel out, together with the first term in Eq. (A3), to obtain Eq. (8). Similar simplifications and their interpretation were discussed already in the case of the Bethe SG [11].

\section{APPENDIX B: RSB EQUATIONS}

Consider the set of coupled equations (14),

$$
\begin{gathered}
\pi(x)=E_{\zeta} \int \prod_{i=1}^{C-1} d \hat{x}_{i} \hat{\pi}(\hat{x}) \delta\left(x-\sum_{i=1}^{C-1} \hat{x}_{i}-F \zeta\right), \\
\hat{\pi}(\hat{x})=E_{J} \int \prod_{j=1}^{K-1} d x_{j} \pi\left(x_{j}\right) \delta\left[\hat{x}-u\left(\left\{x_{j}\right\}, J\right)\right] .
\end{gathered}
$$

For the case of $K=2$, with no random bias, corresponding to the Bethe SG problem, let us define the Laplace transform of the conjugated local field distribution as

$$
g(\sigma)=\int d \hat{x} e^{-\sigma \hat{x}} \hat{\pi}(\hat{x})
$$

Substituting the first equation of Eq. (B1) into the second and then into Eq. (B2), one finds

$$
\begin{aligned}
g(\sigma)= & \int d s \delta(s+i \sigma) E_{J, \zeta} \prod_{j=1}^{C-1} d \hat{x}_{j} \hat{\pi}\left(\hat{x}_{j}\right) \\
& \times \exp \left\{\frac{i}{\beta} \tanh ^{-1}\left[\tanh (\beta J) \tanh \left(\beta \sum_{j=1}^{C-1} \hat{x}_{j}+\beta F\right)\right]\right\}
\end{aligned}
$$

which is the expression for the global order parameter found in Ref. [6] and corresponds to expression (18) for $K=2$. The expression for arbitrary $K$ values of the multispin interaction simply involves multiple convolutions of the Mottishaw global order parameter. The meaning of the global order parameter (B2) can be understood observing that, e.g., in the case of the Bethe SG considered here (in a uniform magnetic field), the partition function is given by [5]

$$
\left\langle Z^{n}\right\rangle \equiv \operatorname{Tr}_{\left\{\sigma_{o}^{\alpha}\right\}} \exp \left(\beta F \sum_{\alpha=1}^{n} \sigma_{o}^{\alpha}\right)\left[g_{m}\left(\sigma_{o}^{\alpha}\right)\right]^{C},
$$

where

$$
\begin{aligned}
g\left(\left\{\sigma_{o}^{\alpha}\right\}\right)= & E_{J}\left[\operatorname{Tr}_{\left\{\sigma_{1}^{\alpha}\right\}}\left(g\left(\left\{\sigma_{1}^{\alpha}\right\}\right)\right)^{C-1}\right. \\
& \left.\times \exp \left(\beta J \sum_{\alpha} \sigma_{1}^{\alpha} \sigma_{o}^{\alpha}+\beta F \sum_{\alpha} \sigma_{1}^{\alpha}\right)\right] .
\end{aligned}
$$

The subscript $m$ indicates the dependence of the global order parameter on the subshell tree structure. If one is interested in the thermodynamical limit, it is expected that the fixed point of the above recursion determines the equilibrium properties of the system. A delicate point is the choice of the boundary conditions which are well known [11] to play an important role in this problem. The corresponding extension of Eq. (B5) to the case of arbitrary $K$ values has been reported in Eq. (17).
[1] Y. Imry and S. Ma, Phys. Rev. Lett. 35, 1399 (1975).

[2] S. F. Edwards and P. W. Anderson, J. Phys. F: Met. Phys. 5, 965 (1975).

[3] K. Wong and D. Sherrington, J. Phys. A 20, L785 (1987).

[4] M. Mézard and G. Parisi, Europhys. Lett. 3, 1067 (1987).

[5] P. Mottishaw, Europhys. Lett. 4, 333 (1987).

[6] P. Mottishaw and C. DeDominicis, J. Phys. A 20, L375 (1987); C. DeDominicis and P. Mottishaw, ibid. 20, L841 (1987).

[7] C. DeDominicis and Y. Y. Goldschmidt, J. Phys. A 22, 399 (1989); Y. Y. Goldschmidt and C. DeDominicis, Phys. Rev. B 41, 2184 (1990).

[8] H. Rieger and T. R. Kirkpatrick, Phys. Rev. B 45, 9772 (1992).

[9] R. Monasson, J. Phys. A 31, 513 (1998).

[10] Y. Y. Goldschmidt and P. Y. Lai, J. Phys. A 23, L775 (1990).

[11] M. Mézard and G. Parisi, Eur. Phys. J. B 20, 217 (2001); J. Stat. Phys. 111, 1 (2003).

[12] M. Mézard, G. Parisi, and R. Zecchina, Science 297, 812 (2002).

[13] R. C. Dewar and P. Mottishaw, J. Phys. A 21, L1135 (1988).
[14] G. Migliorini and A. N. Berker, Phys. Rev. B 57, 426 (1998).

[15] T. Castellani, F. Krzakala, and F. Ricci-Tersenghi, Eur. Phys. J. B 47, 99 (2005).

[16] R. G. Gallager, IRE Trans. Inf. Theory IT-8, 21 (1962); Low Density Parity Check Codes, Research Monograph Series No. 21, (MIT Press, Cambridge, MA, 1963).

[17] C. E. Shannon, Bell Syst. Tech. J. 27, 379 (1948); 27, 623 (1948).

[18] T. Cover and J. Thomas, Elements of Information Theory (Wiley, New York, 1991).

[19] N. Sourlas, Nature (London) 339, 693 (1989).

[20] Y. Kabashima and D. Saad, Europhys. Lett. 45, 97 (1999).

[21] R. Vicente, D. Saad, and Y. Kabashima, Phys. Rev. E 60, 5352 (1999).

[22] A. Montanari, Eur. Phys. J. B 23, 121 (2001).

[23] B. Derrida, Phys. Rev. B 24, 2613 (1981).

[24] K. Nakamura, Y. Kabashima, and D. Saad, Europhys. Lett. 56, 610 (2001).

[25] D. J. C. MacKay, IEEE Trans. Inf. Theory 45, 399 (1999).

[26] Advanced Mean Field Methods-Theory and Practice, edited by M. Opper and D. Saad (MIT Press, Cambridge, MA, 2001). 
[27] J. S. Yedida, W. T. Freeman, and Y. Weiss, Advances in Neural Information Processing Systems, edited by T. K. Leen, T. G. Dietterich, and V. Treps (MIT Press, Cambridge, MA, 2001), Vol. 13.

[28] H. Nishimori, J. Phys. C 13, 4071 (1980).

[29] H. Nishimori, J. Phys. Soc. Jpn. 62, 2973 (1993).

[30] Y. Iba, J. Phys. A 32, 3875 (1999).

[31] S. Franz, M. Leone, A. Montanari, and F. Ricci-Tersenghi, Phys. Rev. E 66, 046120 (2002).

[32] Y. Kabashima and D. Saad, J. Phys. A 37, R1 (2004).

[33] D. R. Bowman and K. Levin, Phys. Rev. B 25, R3438 (1982).

[34] M. Mézard, G. Parisi, and M. Virasoro, Spin Glass Theory and Beyond (World Scientific, Singapore, 1987).

[35] T. Murayama, Y. Kabashima, D. Saad, and R. Vicente, Phys. Rev. E 62, 1577 (2000).

[36] T. Tanaka and D. Saad, J. Phys. A 36, 11143 (2003).

[37] F. Kschischang and B. Frey, IEEE J. Sel. Areas Commun. 2, 153 (1998).

[38] J. Pearl, Probabilistic Reasoning in Intelligent Systems, (Morgan Kaufmann, San Francisco, 1988).

[39] D. Andelman and A. N. Berker, Phys. Rev. B 29, 2630 (1984).

[40] M. Hinczewski and A. N. Berker, Phys. Rev. B 72, 144402 (2005).

[41] A. N. Berker and S. R. McKay, Phys. Rev. B 33, 4712 (1986).

[42] M. Kaufman and R. B. Griffiths, Phys. Rev. B 24, R496 (1981); R. B. Griffiths and M. Kaufman, ibid. 26, 5022
(1982).

[43] I. Kanter and D. Saad, J. Phys. A 33, 1675 (2000).

[44] T. Richardson and R. Urbanke, IEEE Trans. Inf. Theory 47, 599 (2001).

[45] S. R. McKay, A. N. Berker, and S. Kirkpatrick, Phys. Rev. Lett. 48, 767 (1982).

[46] C. Amoruso and A. K. Hartmann, Phys. Rev. B 70, 134425 (2005)

[47] J. van Mourik, D. Saad, and Y. Kabashima, Phys. Rev. E 66, 026705 (2002).

[48] Y. Kabashima, J. Phys. Soc. Jpn. 74, 2133 (2005).

[49] S. Franz, M. Leone, F. Ricci-Tersenghi, and R. Zecchina, Phys. Rev. Lett. 87, 127209 (2001).

[50] M. Mézard and R. Zecchina, Phys. Rev. E 66, 056126 (2002).

[51] M. G. Luby, M. Mitzenmacher, M. A. Shokrollahi, and D. A. Spielman, IEEE Trans. Inf. Theory 47, 569 (2001).

[52] T. Richardson and R. Urbanke, Codes, Systems, and Graphical Models, edited by B. Marcus and J. Rosenthal (Springer, New York, 2001).

[53] C. Measson, A. Montanari, and R. Urbanke (unpublished).

[54] H. Nishimori and D. Sherrington, Disordered and Complex Systems, edited by P. Sollich, A. Coolen, L. Hughston, and R. Streater (AIP, Melville, NY, 2001), p. 67;P. Le Doussal and A. B. Harris, Phys. Rev. Lett. 61, 625 (1988).

[55] D. J. C. MacKay and K. Nakamura (unpublished). 